\title{
ANALYSIS OF WETTING AND CONTACT ANGLE HYSTERESIS ON CHEMICALLY PATTERNED SURFACES*
}

\author{
XIANMIN $\mathrm{XU}^{\dagger}$ AND XIAOPING WANG $\ddagger$
}

\begin{abstract}
Wetting and contact angle hysteresis on chemically patterned surfaces in two dimensions are analyzed from a stationary phase-field model for immiscible two phase fluids. We first study the sharp-interface limit of the model by the method of matched asymptotic expansions. We then justify the results rigorously by the $\Gamma$-convergence theory for the related variational problem and study the properties of the limiting minimizers. The results also provide a clear geometric picture of the equilibrium configuration of the interface. This enables us to explicitly calculate the total surface energy for the two phase systems on chemically patterned surfaces with simple geometries, namely the two phase flow in a channel and the drop spreading. By considering the quasi-static motion of the interface described by the change of volume (or volume fraction), we can follow the change-of-energy landscape which also reveals the mechanism for the stick-slip motion of the interface and contact angle hysteresis on the chemically patterned surfaces. As the interface passes through patterned surfaces, we observe not only stick-slip of the interface and switching of the contact angles but also the hysteresis of contact point and contact angle. Furthermore, as the size of the pattern decreases to zero, the stick-slip becomes weaker but the hysteresis becomes stronger in the sense that one observes either the advancing contact angle or the receding contact angle (when the interface is moving in the opposite direction) without the switching in between.
\end{abstract}

Key words. contact angle hysteresis, phase-field model, $\Gamma$-convergence

AMS subject classifications. 41A60, 49J45, 76T10

DOI. $10.1137 / 110829593$

1. Introduction. The study of the wetting phenomenon is of critical importance for many applications and has attracted much interest in physics and applied mathematics communities, stimulated by the development of surface engineering and by studies on the superhydrophobicity property in a variety of natural and artificial objects $[1,2,10,8,19]$. The primary parameter that characterizes wetting is the static contact angle, which is defined as the measurable angle that a liquid makes with a solid. The contact angle of liquid with a flat, homogeneous surface is given by Young's equation [26],

$$
\cos \theta=\frac{\gamma_{S V}-\gamma_{S L}}{\gamma}
$$

where $\gamma_{S V}, \gamma_{S L}$, and $\gamma$ are the surface tension of the solid-vapor interface, the solidliquid interface, and the liquid-vapor interface, respectively. If the liquid wets the surface (referred to as wetting liquid or hydrophilic surface), the value of the static contact angle is $0^{\circ} \leq \theta \leq 90^{\circ}$, whereas if the liquid does not wet the surface (referred to as nonwetting liquid or hydrophobic surface), the value of the contact angle is

${ }^{*}$ Received by the editors April 4, 2011; accepted for publication (in revised form) June 24, 2011; published electronically September 27, 2011. This work was supported in part by award SA-C0040/UK-C0016, made by King Abdullah University of Science and Technology (KAUST), by Hong Kong RGC-GRF grants 603107 and 604209, and by the National Basic Research Program of China under project 2009CB623200.

http://www.siam.org/journals/siap/71-5/82959.html

${ }^{\dagger}$ Institute of Computational Mathematics and Scientific/Engineering Computing, Chinese Academy of Sciences, Beijing 100080, China (xmxu@lsec.cc.ac.cn). This author's work was supported by NSFC project 11001260 and by the State Key Laboratory of Scientific and Engineering Computing of China.

$\ddagger$ Department of Mathematics, the Hong Kong University of Science and Technology, Clear Water Bay, Kowloon, Hong Kong, China (mawang@ust.hk). 
$90^{\circ} \leq \theta \leq 180^{\circ}$. Surfaces with a contact angle between $150^{\circ}$ and $180^{\circ}$ are called superhydrophobic.

Experimentally, the contact angle of a drop on a rough surface has been observed to take a range of values. The highest (lowest) stable contact angle is termed the advancing (receding) angle $\theta_{a}\left(\theta_{r}\right)$. The observed contact angle is usually velocity dependent, and the advancing (receding) contact angle is defined in the limit of contact line velocity $U \rightarrow 0$ with $U>0(U<0)$ [9]. The contact angle hysteresis $(\mathrm{CAH})$ $\Delta \theta=\theta_{a}-\theta_{r}$ proves to be an important quantity that determines many properties of the surface. The origin of CAH is attributed to several factors such as surface roughness or chemical contaminants [13, 21, 24]. Theoretical models of CAH have focused on roughness and heterogeneity as providing energy barriers for the system to attain the global minimum $[8,20]$.

In this paper, we study the wetting and hysteresis on chemically patterned surfaces based on a phase-field model $((2.8),(2.9))$ and the related variational problem. Using the method of matched asymptotic expansion, we first study the sharp-interface limit of the solution to the phase-field model. We show that the limit describes an interface with constant curvature that intersects the patterned surface with a contact angle $\theta_{s}$. At the border point between the strips, the value of the contact angle is not unique. The asymptotic results can be justified rigorously by the $\Gamma$-convergence theory for the related variational problem and the careful study of the sharp-interface energy minimization problem. The $\Gamma$-convergence theory for chemically homogeneous surfaces has been developed in $[14,15]$. We generalize the results to the case with chemically inhomogeneous surface energy density and prove the convergence of the minimizers of the diffuse-interface problem to those of the sharp-interface problem. We also study the geometric property of the minimizers of the sharp-interface model. The result, which is a generalization of the result in [6] with zero surface energy, justifies what we have obtained by the asymptotic expansions.

Based on the sharp-interface model, we then give a detailed investigation of the stick-slip motion of the interface and the $\mathrm{CAH}$ for two cases with chemically patterned surfaces, namely the quasi-static motion of the two phase flow in a channel and the quasi-static spreading of a liquid drop. By considering motion of the interface as the volume of the drop (or volume fraction of the two fluids) is quasi-statically increased or decreased, one can explicitly compute the energy functions in both cases. As the interface passes through patterned surfaces, we observe not only the stick-slip of the interface and the switching of the contact angles but also the hysteresis of contact point and contact angle. Furthermore, as the size of the pattern decreases to zero, the stick-slip becomes weaker, but the hysteresis becomes stronger in the sense that one observes either the advancing contact angle or the receding contact angle (when the interface moves in the opposite direction) without the switching in between.

We remark that dynamic hysteresis with finite interface speed (instead of quasistatic) was studied numerically in a recent work [22]. We simulated the moving contact line in a two-dimensional chemically patterned channel using a diffuse-interface model with the generalized Navier boundary condition developed in [18]. The motion of the fluid-fluid interface in confined immiscible two phase flows is modulated by the chemical pattern on the top and bottom surfaces, leading to a stick-slip behavior of the contact line. The advancing and receding contact angles are equal to the maximum and minimum values, respectively, of static contact angles of the surfaces. A critical value of the wettability contrast is identified, above which the effect of diffusion becomes important, leading to the interesting behavior of fluid-fluid interface breaking, with the transport of the nonwetting fluid being assisted and mediated by 
rapid diffusion through the wetting fluid. Near the critical value, the time-averaged extra dissipation scales as $U$, the averaged displacement velocity.

The paper is organized as follows. In section 2, we introduce the stationary two phase fluid model. We then carry out matched asymptotic analysis for the sharpinterface limit in section 3 . In section 4 , we state and prove the $\Gamma$-convergence theorem for the sharp-interface limit. In section 5 , we consider two explicit examples and study the stick-slip jump of the interface and the $\mathrm{CAH}$.

2. The stationary two phase fluid model. In immiscible two phase flows, the contact line denotes the intersection of the fluid-fluid interface with the solid wall. It has been shown that the no-slip boundary condition leads to nonphysical contactline singularity, or infinite viscous dissipation near the moving contact line. Recently, a diffuse-interface model with generalized Navier boundary condition was proposed in [18] to describe the interface motion on solid surfaces, which involves a coupled system of the Navier-Stokes (NS) equation for the velocity field $\mathbf{u}$ in the presence of the capillary force density and the Cahn-Hilliard $(\mathrm{CH})$ equation for the phase field $\phi$ :

$$
\left\{\begin{array}{l}
\mathbf{u}_{t}+(\mathbf{u} \cdot \nabla) \mathbf{u}=-\nabla p+\nu \Delta \mathbf{u}+B \mu \nabla \phi \\
\phi_{t}+\mathbf{u} \cdot \nabla \phi=L_{d} \Delta \mu \\
\operatorname{div} \mathbf{u}=0 \\
\mu=-\epsilon \Delta \phi+f^{\prime}(\phi) / \epsilon
\end{array}\right.
$$

Here $p$ is the pressure, $\mu$ is the chemical potential, and $f(\phi)$ is the bulk free energy density. $\nu, B, L_{d}$ are physical parameters, and $\epsilon$ is a small parameter to characterize the width of the interface between the two fluids. In general, $f(\cdot)$ is a double well function. For simplicity, we choose it as $f(\phi)=\frac{\left(1-\phi^{2}\right)^{2}}{4}$. In this case, the states $\phi= \pm 1$ represent the two phases of the fluid, respectively.

The boundary conditions at the solid surface are the impermeability conditions $\partial_{n} \mu=0$ and $v_{n}=0$, the relaxational equation for $\phi$,

$$
\frac{\partial \phi}{\partial t}+u_{\tau} \partial_{\tau} \phi=-V_{s} L(\phi)
$$

and the generalized Navier boundary condition

$$
L_{s}^{-1} u_{\tau}^{s l i p}=-\partial_{n} u_{\tau}+B \epsilon^{-1} L(\phi) \partial_{\tau} \phi
$$

Here $\tau$ denotes the direction tangent to the solid surface (for two-dimensional flows), $n$ denotes the outward surface normal, $V_{s}$ is a positive phenomenological parameter, $L(\phi)=\epsilon \partial_{n} \phi+\partial \gamma(\mathbf{x}, \phi) / \partial \phi$ with $\gamma(\mathbf{x}, \phi)$ being the fluid-solid interfacial free energy per unit area, $L_{s}$ is the slip length, and $L(\phi) \partial_{\tau} \phi$ is the uncompensated Young stress. Notice that $\gamma(\mathbf{x}, \phi)$ depends on the position $\mathbf{x}$ on the surface, so that it also models the inhomogeneous boundaries.

It is shown that the continuum calculations based on the new model can quantitatively reproduce molecular dynamics simulation results [18], which shows that the new model provides an accurate description of fluid-solid interfacial phenomena, and it also provides an effective tool for performing simulations of two phase flow on rough surfaces.

To describe the stationary wetting phenomena of the fluid on the solid substrates, we let $\mathbf{u}=0$ and $\partial_{t} \phi=0$. Then the NS-CH system (2.1) is reduced to the following system:

$$
\left\{\begin{array}{l}
-\nabla p+B \mu \nabla \phi=0 \\
\Delta \mu=0 \\
\mu=-\epsilon \Delta \phi+f^{\prime}(\phi) / \epsilon
\end{array}\right.
$$

Copyright (c) by SIAM. Unauthorized reproduction of this article is prohibited. 
The boundary conditions become

$$
\left\{\begin{array}{l}
\epsilon \partial_{\mathbf{n}} \phi+\partial_{\phi} \gamma(\mathbf{x}, \phi)=0 \\
\partial_{\mathbf{n}} \mu=0
\end{array}\right.
$$

The stationary NS-CH equations (2.4) and (2.5) could be further simplified. From the equation for chemical potential,

$$
\begin{cases}\Delta \mu=0 & \text { in } \Omega \\ \partial_{\mathbf{n}} \mu=0 & \text { on } \partial \Omega\end{cases}
$$

we have

$$
\mu \equiv c,
$$

with some constant $c$ to be determined. Thus, the equations for $\phi$ and $p$ are reduced to

$$
\begin{cases}-\epsilon \Delta \phi+f^{\prime}(\phi) / \epsilon=c & \text { in } \Omega \\ \epsilon \partial_{\mathbf{n}} \phi+\partial_{\phi} \gamma(\mathbf{x}, \phi)=0 & \text { on } \partial \Omega\end{cases}
$$

and

$$
-\nabla p+B c \nabla \phi=0 \text { in } \Omega .
$$

This is the phase-field model to be studied in this paper.

We remark that (2.8) is the Euler-Lagrange equation of the following constraint functional minimizing problem:

$$
\begin{aligned}
& \min I_{\epsilon}(\phi)=\int_{\Omega} \frac{\epsilon}{2}|\nabla \phi|^{2}+\frac{f(\phi)}{\epsilon} d x d y+\int_{\partial \Omega} \gamma(\mathbf{x}, \phi) d s, \\
& \text { s.t. } \int_{\Omega} \phi=C_{0},
\end{aligned}
$$

with some given constant $C_{0}$ such that $-|\Omega|<C_{0}<|\Omega|$. The constant $c$ in (2.8) is the Lagrange multiplier for the constrained minimization problem.

3. Sharp-interface limit from matched asymptotic expansion. In this section, we will study the behavior of (2.8) and (2.9) when the interface width parameter $\epsilon \rightarrow 0$. We use the matched asymptotic expansion method, which has been successfully used in many related free-interface problems (see, for example, [17, 23]). Here we consider the case when the fluid-fluid interface intersects with a flat but chemically patterned surface. The results obtained by asymptotic analysis will be proved in the next section. From now on, we will use the notation $\phi^{\epsilon}, p^{\epsilon}$, and $c_{\epsilon}$ to indicate the explicit dependence of the respective quantities on the small parameter $\epsilon$.

We assume that the interface is given by the zero level set $\Gamma^{\epsilon}=\{\mathbf{x} \in \bar{\Omega} \mid$ $\left.\phi^{\epsilon}(\mathbf{x})=0\right\}$. Let $d^{\epsilon}(\mathbf{x})$ be the signed distance from the point $\mathbf{x} \in \Omega$ in the neighborhood of $\Gamma^{\epsilon}$ to the interface. The signed distance is such that $\left|\nabla d^{\epsilon}\right|=1$. Suppose that the $d^{\epsilon}(\mathbf{x})$ has the expansion that

$$
d^{\epsilon}(\mathbf{x})=\sum_{j \geq 0} \epsilon^{j} d_{j}(\mathbf{x})
$$

then we have that $d_{0}(\mathbf{x})$ is the leading order spatial signed distance from the point $\mathbf{x} \in \Omega^{ \pm}=\left\{\mathbf{x} \in \bar{\Omega} \mid \pm d^{0}(\mathbf{x})>0\right\}$ to $\Gamma^{0}=\left\{\mathbf{x} \in \bar{\Omega} \mid d_{0}(\mathbf{x})=0\right\}$ and $\left|\nabla d_{0}\right|=1$. The matched asymptotic analysis is based on an outer expansion away from $\Gamma^{0}$ and an inner expansion in the vicinity of $\Gamma^{0}$. 
3.1. The analysis away from the solid boundary. We first study the asymptotic behavior of the solutions away from the solid surface. The analysis in this subsection is standard $[4,5]$.

The outer expansions. We consider the following expansions on $\epsilon$ :

$$
\begin{aligned}
\phi^{\epsilon}(\mathbf{x}) & =\phi_{0}(\mathbf{x})+\epsilon \phi_{1}(\mathbf{x})+\epsilon^{2} \phi_{2}(\mathbf{x})+\cdots, \\
p^{\epsilon}(\mathbf{x}) & =p_{0}(\mathbf{x})+\epsilon p_{1}(\mathbf{x})+\epsilon^{2} p_{2}(\mathbf{x})+\cdots, \\
c_{\epsilon} & =c_{0}+\epsilon c_{1}+\epsilon^{2} c_{2}+\cdots .
\end{aligned}
$$

We substitute the expansions into $(2.8)_{1}$ and (2.9). The leading order of $(2.8)_{1}$ gives

$$
f^{\prime}\left(\phi_{0}\right)=0
$$

Notice that $f^{\prime}(\phi)=-\phi+\phi^{3}$; we have immediately that

$$
\phi_{0}= \pm 1 \quad \text { in } \Omega^{ \pm} .
$$

The leading order of (2.9) gives that

$$
-\nabla p_{0}+B c_{0} \nabla \phi_{0}=0 \quad \text { in } \Omega^{ \pm} .
$$

This implies that $\nabla p_{0}=0$ in $\Omega^{+}$and $\Omega^{-}$or, equivalently,

$$
p_{0}=p_{0}^{ \pm} \quad \text { in } \Omega^{ \pm},
$$

with two constants $p_{0}^{+}$and $p_{0}^{-}$.

The inner expansions. Before we do inner expansions, we first define

$$
\mathbf{m}=\nabla d_{0}(\mathbf{x}), \quad \kappa=\Delta d_{0}(\mathbf{x}) .
$$

When $\mathbf{x} \in \Gamma^{0}, \mathbf{m}$ is the unit normal to $\Gamma^{0}$ pointing toward $\Omega^{+}$, and $\kappa$ is the signed mean curvature of $\Gamma^{0}$ at $\mathbf{x}$, which is positive when the center of the curvature lies in $\Omega^{-}$. We introduce the inner variable in the vicinity of $\Gamma^{0}$,

$$
\xi=d^{0}(\mathbf{x}) / \epsilon
$$

Then we suppose that near $\Gamma^{0}, \phi^{\epsilon}$ and $p^{\epsilon}$ could be written in $(\mathbf{x}, \xi)$ as

$$
\phi^{\epsilon}(\mathbf{x})=\tilde{\phi}(\mathbf{x}, \xi), \quad p^{\epsilon}(\mathbf{x})=\tilde{p}(\mathbf{x}, \xi) .
$$

It is easy to see that

$$
\left.\begin{array}{l}
\nabla=\nabla_{\mathbf{x}}+\epsilon^{-1} \mathbf{m} \partial_{\xi} \\
\Delta=\Delta_{\mathbf{x}}+\epsilon^{-1} \kappa \partial_{\xi}+\epsilon^{-2} \partial_{\xi \xi}
\end{array}\right\}
$$

Thus, $(2.8)_{1}$ and (2.9) could be rewritten as

$$
-\epsilon^{-1} \partial_{\xi \xi} \tilde{\phi}-\kappa \partial_{\xi} \tilde{\phi}-\epsilon \Delta_{\mathbf{x}} \tilde{\phi}+\epsilon^{-1}\left(-\tilde{\phi}+\tilde{\phi}^{3}\right)=c_{\epsilon} \quad \text { in } \Omega
$$

and

$$
\nabla_{\mathbf{x}}\left(-\tilde{p}+B c_{\epsilon} \tilde{\phi}\right)+\epsilon^{-1} \mathbf{m} \partial_{\xi}\left(-\tilde{p}+B c_{\epsilon} \tilde{\phi}\right)=0 \quad \text { in } \Omega
$$

Copyright (c) by SIAM. Unauthorized reproduction of this article is prohibited. 
Now we suppose the following expansions of $\tilde{\phi}$ and $\tilde{p}$ :

$$
\begin{aligned}
& \tilde{\phi}(\mathbf{x}, \xi)=\tilde{\phi}_{0}(\mathbf{x}, \xi)+\epsilon \tilde{\phi}_{1}(\mathbf{x}, \xi)+\epsilon^{2} \tilde{\phi}_{2}(\mathbf{x}, \xi)+\cdots, \\
& \tilde{p}(\mathbf{x}, \xi)=\tilde{p}_{0}(\mathbf{x}, \xi)+\epsilon \tilde{p}_{1}(\mathbf{x}, \xi)+\epsilon^{2} \tilde{p}_{2}(\mathbf{x}, \xi)+\cdots .
\end{aligned}
$$

We substitute these expansions into (3.8) and (3.9). Then we have, for the leading order,

$$
-\partial_{\xi \xi} \tilde{\phi}_{0}+\left(-\tilde{\phi}_{0}+\tilde{\phi}_{0}^{3}\right)=0
$$

and

$$
\partial_{\xi} \tilde{p}_{0}-B c_{0} \partial_{\xi} \tilde{\phi}_{0}=0
$$

In the next order, we have

$$
-\kappa \partial_{\xi} \tilde{\phi}_{0}+\left(-\partial_{\xi \xi} \tilde{\phi}_{1}-\tilde{\phi}_{1}+3 \tilde{\phi}_{0}^{2} \tilde{\phi}_{1}\right)=c_{0} .
$$

Matching the inner and outer expansions. We require the following matching conditions:

$$
\begin{aligned}
& \sum_{j \geq 0} \epsilon^{j} \tilde{\phi}_{j}(\mathbf{x}, \xi) \approx \sum_{j \geq 0} \epsilon^{j} \phi_{j}(\mathbf{x}), \\
& \sum_{j \geq 0} \epsilon^{j} \tilde{p}_{j}(\mathbf{x}, \xi) \approx \sum_{j \geq 0} \epsilon^{j} p_{j}(\mathbf{x}),
\end{aligned}
$$

for $\xi=\frac{d^{0}(\mathbf{x})}{\epsilon}$ near $\Gamma^{0}$. Therefore, we have

$$
\lim _{\xi \rightarrow \pm \infty} \tilde{\phi}_{0}=\phi_{0}^{ \pm}= \pm 1, \quad \lim _{\xi \rightarrow \pm \infty} \tilde{p}_{0}=p_{0}^{ \pm} .
$$

Combining with (3.12) and $\tilde{\phi}_{0}(\mathbf{x}, 0)=0$, we could conclude that

$$
\tilde{\phi}_{0}(\mathbf{x}, \xi)=\Phi(\xi) \quad \forall \mathbf{x} \in \Gamma^{0} \cap \Omega,
$$

where $\Phi$ is the unique solution of the following ordinary differential equation:

$$
\left\{\begin{array}{l}
-\Phi^{\prime \prime}(\xi)-\Phi(\xi)+\Phi(\xi)^{3}=0, \quad-\infty<\xi<+\infty \\
\lim _{\xi \rightarrow \pm \infty} \Phi= \pm 1, \quad \Phi(0)=0 .
\end{array}\right.
$$

Then from (3.14), $\tilde{\phi}_{1}$ is such that

$$
-\partial_{\xi \xi} \tilde{\phi}_{1}-\tilde{\phi}_{1}+3 \Phi^{2} \tilde{\phi}_{1}=c_{0}+\kappa \Phi^{\prime} .
$$

We multiply the equation by $\Phi^{\prime}$ and integrate in $\xi$ from $(-\infty,+\infty)$. The left-hand side then vanishes, and we obtain

$$
2 c_{0}+\kappa \int_{-\infty}^{\infty}\left(\Phi^{\prime}\right)^{2} d \xi=0
$$

Denote the constant $\sigma=\int_{-\infty}^{\infty}\left(\Phi^{\prime}\right)^{2} d \xi$, which represents the interface tension [4]. We are led to

$$
\kappa=-2 c_{0} / \sigma .
$$

Therefore the curvature is a constant.

Furthermore, we integrate (3.13) in $\xi$ from $-\infty$ to $+\infty$, and we have

$$
\left[p_{0}\right]=2 B c_{0}=-B \sigma \kappa,
$$

where $\left[p_{0}\right]=p_{0}^{+}-p_{0}^{-}$is the jump of pressure on the interface $\Gamma^{0}$. This is exactly the Laplace formula in fluid dynamics.

Copyright (C) by SIAM. Unauthorized reproduction of this article is prohibited. 


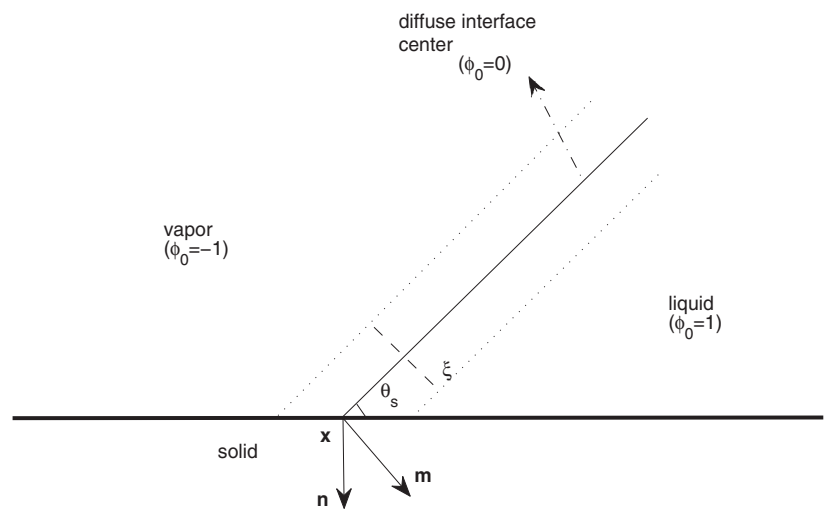

FIG. 3.1. The inner expansion on solid surfaces.

3.2. Analysis on solid boundaries. We now study the asymptotic behavior of (2.8) on solid boundaries. We first consider the outer expansion away from the contact point. Substituting the expansion (3.1) into $(2.8)_{2}$, the leading order then leads to

$$
\partial_{\phi} \gamma\left(\mathbf{x}, \phi_{0}\right)=0 \quad \text { on } \partial \Omega \cap \Omega^{ \pm} .
$$

Notice that $\phi_{0}= \pm 1$ in the field $\Omega^{ \pm}$, respectively. So we have

$$
\begin{aligned}
\partial_{\phi} \gamma(\mathbf{x}, 1)=0, & \mathbf{x} \in \partial \Omega \cap \Omega^{+}, \\
\partial_{\phi} \gamma(\mathbf{x},-1)=0, & \mathbf{x} \in \partial \Omega \cap \Omega^{-} .
\end{aligned}
$$

We then consider the inner expansion near the contact point on the solid boundary. Using the local coordinate $\xi$ defined in the last subsection, $(2.8)_{2}$ then reads

$$
\epsilon \mathbf{n} \cdot\left(\nabla_{\mathbf{x}}+\epsilon^{-1} \mathbf{m}\right) \partial_{\xi} \tilde{\phi}+\partial_{\phi} \gamma(\mathbf{x}, \tilde{\phi})=0 \quad \text { on } \partial \Omega .
$$

Here $\mathbf{n}$ is the out unit normal of the boundary $\partial \Omega$, and $\mathbf{m}$ is the unit normal of the interface $\Gamma$ pointing into $\Omega^{+}$(see Figure 3.1). Then we substitute the inner expansion (3.10) into the equation and take the leading order, obtaining

$$
\mathbf{n} \cdot \mathbf{m} \partial_{\xi} \tilde{\phi}_{0}+\partial_{\phi} \gamma\left(\mathbf{x}, \tilde{\phi}_{0}\right)=0 \quad \text { on } \partial \Omega .
$$

We suppose that the formula (3.16) on $\tilde{\phi}_{0}$ could be extended directly to the boundary $\partial \Omega$ by keeping the values invariant along the tangential direction of the interface $\Gamma$ (as shown in Figure 3.1). Then from the above equation, we know that

$$
\mathbf{n} \cdot \mathbf{m} \Phi^{\prime}+\partial_{\phi} \gamma(\mathbf{x}, \Phi)=0
$$

for the contact point $\mathbf{x} \in \Gamma \cap \partial \Omega$. Here $\Phi$ is given by (3.17).

We assume that the two domains $\Omega^{ \pm}$where $\phi_{0}=+1$ and $\phi_{0}=-1$ represent the liquid phase and the vapor phase, respectively. It is easy to see that $\mathbf{n} \cdot \mathbf{m}=\cos \theta_{s}$, with $\theta_{s}$ the static contact angle of the liquid phase $\left(\phi_{0}=1\right)$ on the solid boundary. So the above equation could be rewritten as

$$
\Phi^{\prime} \cos \theta_{s}+\partial_{\phi} \gamma(\mathbf{x}, \Phi)=0 .
$$


The chemically homogeneous surface. When the solid interface is homogeneous, i.e., $\gamma(\mathbf{x}, \Phi)$ is independent of $\mathbf{x}$ and $\gamma(\mathbf{x}, \Phi)=\gamma(\Phi)$, then we multiply (3.25) by $\Phi^{\prime}$ and integrate in $\xi$ from $-\infty$ to $+\infty$, obtaining

$$
\int_{-\infty}^{+\infty}\left(\Phi^{\prime}\right)^{2} d \xi \cos \theta_{s}+\int_{-\infty}^{+\infty} \gamma^{\prime}(\Phi) \Phi^{\prime} d \xi=0
$$

or, equivalently,

$$
\int_{-\infty}^{+\infty}\left(\Phi^{\prime}\right)^{2} d \xi \cos \theta_{s}+\int_{-1}^{1} \gamma^{\prime}(\Phi) d \Phi=0
$$

This gives

$$
\sigma \cos \theta_{s}+(\gamma(1)-\gamma(-1))=0
$$

with $\sigma=\int_{-\infty}^{+\infty}\left(\Phi^{\prime}\right)^{2} d \xi$ being the surface tension. We denote $\gamma_{S V}=\gamma(-1)$ and $\gamma_{S L}=\gamma(1)$, which represent the solid-vapor and solid-liquid surface energy density, respectively. Then the above equation is

$$
\cos \theta_{s}=\frac{\gamma_{S V}-\gamma_{S L}}{\sigma}
$$

which is the so-called Young's equation on wetting contact angles.

For simplicity, we assume that $\gamma(\phi)$ is a function interpolating $\gamma_{S V}=\gamma(-1)$ and $\gamma_{S L}=\gamma(1)$ in the form of $\gamma(\phi)=\frac{\gamma_{S V}+\gamma_{S L}}{2}-\frac{\gamma_{S V}-\gamma_{S L}}{4}\left(3 \phi-\phi^{3}\right)$. (This is the unique formula for $(3.25)$ to be exactly correct on a planar surface [25, 27].) Then from the Young's equation (3.27), we have

$$
\gamma^{\prime}(\phi)=-\frac{\sigma}{2} \cos \theta_{s} s_{\gamma}(\phi)
$$

with $s_{\gamma}=\frac{3}{2}\left(1-\phi^{2}\right)$. It is easy to see that such a formula for $\gamma^{\prime}(\phi)$ satisfies (3.21) and (3.22).

The chemically patterned surface. We assume that the solid boundary is periodically patterned with two parts $\mathcal{A}$ and $\mathcal{B}$ with different surface energy densities $\gamma_{\mathcal{A}}(\phi)$ and $\gamma_{\mathcal{B}}(\phi)$ such that

$$
\gamma_{\mathcal{A}}^{\prime}(\phi)=-\frac{\sigma}{2} \cos \theta_{\mathcal{A}} s_{\gamma}(\phi), \quad \gamma_{\mathcal{B}}^{\prime}(\phi)=-\frac{\sigma}{2} \cos \theta_{\mathcal{B}} s_{\gamma}(\phi),
$$

with different angles $\theta_{\mathcal{A}}$ and $\theta_{\mathcal{B}}$. We then define the surface energy density $\gamma(\mathbf{x}, \phi)$ on each period as

$$
\gamma(\mathbf{x}, \phi)= \begin{cases}\gamma_{\mathcal{A}}(\phi), & \mathbf{x} \in \mathcal{A} \\ \gamma_{\mathcal{B}}(\phi), & \mathbf{x} \in \mathcal{B}\end{cases}
$$

Here $\mathcal{A}$ and $\mathcal{B}$ are disjoint open sets. We denote $\mathcal{R}$ as the set of discrete border points between $\mathcal{A}$ and $\mathcal{B}$.

Let $\tilde{\mathbf{x}}_{0}$ be a contact point (the intersection of the interface with the boundary). Similar to the derivation for the chemically homogeneous boundary, when $\tilde{\mathbf{x}}_{0} \in \mathcal{A}$ we have

$$
\theta_{s}=\theta_{\mathcal{A}}
$$

Copyright ( $\odot$ by SIAM. Unauthorized reproduction of this article is prohibited. 


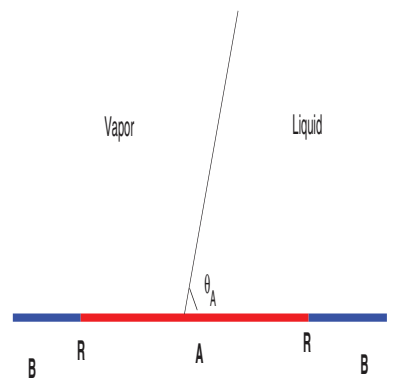

(a) The contact point on $\mathcal{A}$.

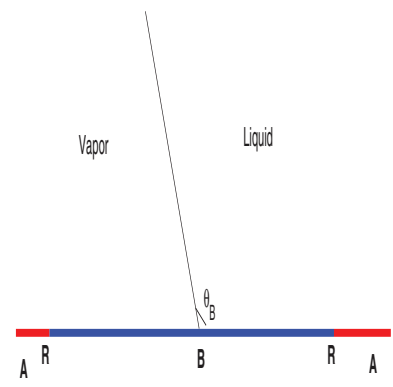

(b) The contact point on $\mathcal{B}$.

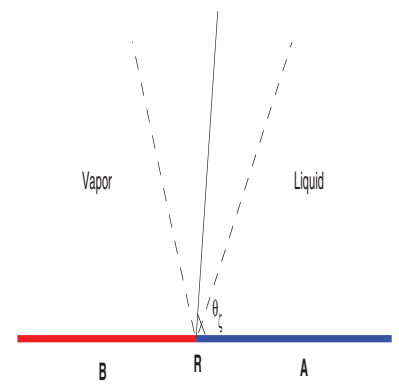

(c) The contact point at $\mathcal{R}$.

FIG. 3.2. The contact angles on a chemically patterned surface.

and when $\tilde{\mathbf{x}}_{0} \in \mathcal{B}$ we have

$$
\theta_{s}=\theta_{\mathcal{B}}
$$

The situation is more complicated when the contact point $\tilde{\mathbf{x}}_{0}$ is the common boundary point $\mathbf{x}_{0}$ defined above, since the surface energy density function $\gamma(\mathbf{x}, \phi)$ is discontinuous at $\mathbf{x}_{0}$. To perform the asymptotic analysis in this case, we smooth out $\gamma(\mathbf{x}, \phi)$ as follows:

$$
\gamma^{\delta}(\mathbf{x}, \phi)= \begin{cases}\gamma_{\mathcal{A}}(\phi), & s\left(\mathbf{x}, \mathbf{x}_{0}\right)<-\frac{\delta}{2}, \\ \gamma_{\mathcal{B}}(\phi), & s\left(\mathbf{x}, \mathbf{x}_{0}\right)>\frac{\delta}{2}, \\ \left(\frac{1}{2}+\frac{s\left(\mathbf{x}, \mathbf{x}_{0}\right)}{\delta}\right) \gamma_{\mathcal{A}}(\phi)+\left(\frac{1}{2}-\frac{s\left(\mathbf{x}, \mathbf{x}_{0}\right)}{\delta}\right) \gamma_{\mathcal{B}}(\phi), & -\frac{\delta}{2} \leq s\left(\mathbf{x}, \mathbf{x}_{0}\right) \leq \frac{\delta}{2} .\end{cases}
$$

Here $s\left(\mathbf{x}, \mathbf{x}_{0}\right)$ is the signed distance from $\mathbf{x}$ to $\mathbf{x}_{0}$ along the solid boundary, and $\delta>0$ is a small parameter. We now assume that the contact point $\tilde{\mathbf{x}}_{0}$ is in the small neighborhood of $\mathbf{x}_{0}$ such that $-\frac{\delta}{2} \leq s\left(\tilde{\mathbf{x}}_{0}, \mathbf{x}_{0}\right) \leq \frac{\delta}{2}$. Then from (3.25), we have

$$
\int_{-\infty}^{+\infty}\left(\Phi^{\prime}\right)^{2} d \xi \cos \theta_{s}+\lambda \int_{-\infty}^{+\infty} \gamma_{\mathcal{A}}^{\prime}(\Phi) \Phi^{\prime} d \xi+(1-\lambda) \int_{-\infty}^{+\infty} \gamma_{\mathcal{B}}^{\prime}(\Phi) \Phi^{\prime} d \xi=0,
$$

where $\lambda=\frac{1}{2}+\frac{s\left(\tilde{\mathbf{x}_{0}}, \mathbf{x}_{0}\right)}{\delta} \in[0,1]$. This implies that the contact angle on $\tilde{\mathbf{x}}_{0}$ is such that

$$
\cos \theta_{s}=\lambda \cos \theta_{\mathcal{A}}+(1-\lambda) \cos \theta_{\mathcal{B}}
$$

Then let $\delta \rightarrow 0, s\left(\tilde{\mathbf{x}}_{0}, \mathbf{x}_{0}\right) \rightarrow 0$ while $s\left(\tilde{\mathbf{x}}_{0}, \mathbf{x}_{0}\right) / \delta$ is kept fixed. We get that the contact angle on $\mathbf{x}_{0}$ could be given by

$$
\cos \theta_{s}=\lambda \cos \theta_{\mathcal{A}}+(1-\lambda) \cos \theta_{\mathcal{B}} .
$$

Notice that $\lambda$ can take any value between 0 and 1 under various choice of $\tilde{\mathbf{x}}_{0}$ in the above analysis. Therefore, when the contact point is exactly the meeting point between $\mathcal{A}$ and $\mathcal{B}$, the contact angle $\theta_{s}$ is not unique and can take any value in the range $\theta_{\mathcal{A}} \leq \theta_{s} \leq \theta_{\mathcal{B}}$. The exact value of $\theta_{s}$ has to be determined by other conditions. In the above analysis, it depends on how $\tilde{\mathbf{x}}_{0}$ approaches $\mathbf{x}_{0}$.

In conclusion, on a chemically patterned surface, we have the contact angle at the contact point $\tilde{\mathbf{x}}_{0}$ in various situations as (see Figure 3.2)

$$
\theta_{s}= \begin{cases}\theta_{\mathcal{A}} & \text { if } \tilde{\mathbf{x}}_{0} \in \mathcal{A} \\ \theta_{\mathcal{B}} & \text { if } \tilde{\mathbf{x}}_{0} \in \mathcal{B} \\ \theta_{\zeta} & \text { if } \tilde{\mathbf{x}}_{0} \in \mathcal{R}\end{cases}
$$

Copyright $@$ by SIAM. Unauthorized reproduction of this article is prohibited. 
with some $\theta_{\zeta}$ such that $\theta_{\mathcal{A}} \leq \theta_{\zeta} \leq \theta_{\mathcal{B}}$. At the borders of the patterned strips, the value of the contact angle is not unique and has to be determined by additional physical conditions.

4. Analysis of the variational problem. The results by asymptotic analysis in the last section will be proved in this section. It is easy to see that the stationary problem (2.8) is equivalent to the following energy minimizing problem:

$$
\min I_{\epsilon}\left(\phi^{\epsilon}\right)= \begin{cases}\int_{\Omega} \frac{\epsilon}{2}\left|\nabla \phi^{\epsilon}\right|^{2}+\frac{f\left(\phi^{\epsilon}\right)}{\epsilon} d x d y+\int_{\partial \Omega} \gamma\left(\mathbf{x}, \phi^{\epsilon}\right) d s & \text { if } \int_{\Omega} \phi^{\epsilon}=C_{0}, \\ +\infty & \text { otherwise, }\end{cases}
$$

with some fixed constant $-|\Omega|<C_{0}<|\Omega|$. Thus, to understand the behavior of the stable solution of (2.8), it is equivalent to study the minimizers of the problem (4.1).

4.1. Preliminary. The convergence of functional minimizing problems could be illustrated through $\Gamma$-convergence theory $[3,7]$. In this subsection, we introduce some general definitions and results on $\Gamma$-convergence. The following definition of $\Gamma$-convergence is given in [3].

DeFINITION 4.1. Let $U$ be a metric space equipped with the distance $\rho$. We say that a sequence of functionals $F_{j}: U \rightarrow \mathbb{R} \cup\{\infty\} \Gamma$-converges to $F_{0}: U \rightarrow \mathbb{R} \cup\{\infty\}$ if for all $u \in U$ we have

(i) (lower bound inequality) for every sequence $u_{j}$ converging to $u$

$$
F_{0}(u) \leq \liminf _{j \rightarrow \infty} F_{j}\left(u_{j}\right)
$$

(ii) (upper bound inequality) there exists a sequence $u_{j}$ converging to $u$ such that

$$
F_{0}(u) \geq \limsup _{j \rightarrow \infty} F_{j}\left(u_{j}\right) .
$$

We then introduce some definitions on local minimizers [14]. We say that $u$ is a local minimizer of a functional $F$ if

$$
F(u) \leq F(v) \quad \text { whenever } \rho(u, v)<\delta
$$

for some $\delta>0 . u$ is called an isolated local minimizer of the functional $F$ if

$$
F(u)<F(v) \quad \text { whenever } 0<\rho(u, v)<\delta
$$

for some $\delta>0$. Specifically, we say that $u$ is an $L_{1}$-local minimizer (an isolated $L_{1}$-local minimizer $)$ if the distance $\rho$ in the above definitions is given by $\rho(u, v)=$ $\|u-v\|_{L^{1}(\Omega)}$.

To study the convergence behavior of minimizers based on $\Gamma$-convergence theory, we need more restrictions on the functionals. A functional $F$ is said to be lower semicontinuous if, for any $u \in U$ and any sequence $u_{j}$ converging to $u$, we have

$$
F(u) \leq \liminf _{j \rightarrow \infty} F\left(u_{j}\right) .
$$

A sequence of functionals $F_{j}$ is said to be equicoercive if for any sequence $u_{j}$ such that $\sup _{j} F_{j}\left(u_{j}\right)<\infty$ there exists a subsequence of $u_{j}$ converging to some $u \in U$.

The following lemma is given by Kohn and Sternberg [14].

Lemma 4.1. Let $(U, \rho)$ be a complete metric space, and consider a sequence of functionals $F_{j}: U \rightarrow \mathbb{R} \cup\{\infty\}$. Suppose that the latter are equicoercive and lower semicontinuous. Suppose also that the sequence $F_{j} \Gamma$-converges to $F_{0}$ as $j \rightarrow \infty$. If $u_{0}$ is an isolated local minimizer of $F_{0}$, then there is a sequence $\left\{u_{j}\right\}$ of local minimizers of $F_{j}$ such that $\rho\left(u_{j}, u_{0}\right) \rightarrow 0$ as $j \rightarrow \infty$. 
4.2. The $\Gamma$-convergence result. We now study the limiting behavior of the problem (4.1) as $\epsilon$ goes to 0. A similar problem with chemically homogeneous boundary has been analyzed by Modica [15]. The following proposition is a direct generalization of the result in [15].

Proposition 4.1. In the bounded variational function space $B V(\Omega)$, the functional $I_{\epsilon} \Gamma$-converges to $\tilde{I}_{0}$ in the $L^{1}(\Omega)$ sense as $\epsilon$ goes to 0 , with $\tilde{I}_{0}$ being defined as

$$
\tilde{I}_{0}(\phi)=\left\{\begin{array}{lr}
\tilde{\sigma}\left|\partial \Omega_{1} \cap \Omega\right|+\int_{\partial \Omega_{1} \cap \partial \Omega} \tilde{\gamma}(\mathbf{x}, 1) d s+\int_{\partial \Omega \backslash \partial \Omega_{1}} \tilde{\gamma}(\mathbf{x},-1) d s & \text { if } \phi(x)= \pm 1 \text { a.e. } \\
+\infty & \text { and } \int_{\Omega} \phi=C_{0} \\
\text { otherwise. }
\end{array}\right.
$$

Here $\Omega_{1}=\{\mathbf{x} \in \Omega: \phi(\mathbf{x})=1\}$, the surface energy density is

$$
\tilde{\gamma}(\mathbf{x}, t)=\inf _{s}\left\{\gamma(\mathbf{x}, s)+\left|\int_{s}^{t}(2 f(r))^{1 / 2} d r\right|\right\}
$$

and the interface energy density is

$$
\tilde{\sigma}=\int_{-1}^{1}(2 f(r))^{1 / 2} d r
$$

Remark 4.1. The difference of the above result from that of Modica [15] is that we allow an inhomogeneous boundary energy density $\gamma(\mathbf{x}, \phi)$. The proof of the proposition is similar to that of Theorem $2.1 \mathrm{in} \mathrm{[15]} \mathrm{and} \mathrm{is} \mathrm{given} \mathrm{in} \mathrm{the} \mathrm{appendix.}$

For a specific choice of the bulk free energy density $f(\phi)=\frac{\left(1-\phi^{2}\right)^{2}}{4}$, we have the following proposition.

Proposition 4.2. In the bounded variational function space $B V(\Omega)$, the functional $I_{\epsilon} \Gamma$-converges to $I_{0}$ in the $L^{1}(\Omega)$ sense as $\epsilon$ goes to 0 , with $I_{0}$ being defined as

$$
I_{0}(\phi)=\left\{\begin{array}{lr}
\sigma\left|\partial \Omega_{1} \cap \Omega\right|+\int_{\partial \Omega_{1} \cap \partial \Omega} \gamma(\mathbf{x}, 1) d s+\int_{\partial \Omega \backslash \partial \Omega_{1}} \gamma(\mathbf{x},-1) d s & \text { if } \phi(x)= \pm 1 \text { a.e. } \\
+\infty & \text { and } \int_{\Omega} \phi=C_{0} \\
\text { otherwise. }
\end{array}\right.
$$

Here $\Omega_{1}=\{x \in \Omega: \phi(x)=1\}$, and the interface energy density is given by

$$
\sigma=\int_{-\infty}^{+\infty}\left(\Phi^{\prime}\right)^{2} d \xi
$$

with $\Phi(\xi)$ defined by (3.17).

Proof. We need to show only that $I_{0}$ is the same as $\tilde{I}_{0}$ under the special choice of $f(\phi)=\frac{\left(1-\phi^{2}\right)^{2}}{4}$.

First, we show that $\sigma=\tilde{\sigma}$. Notice that from (3.17) we have

$$
\Phi^{\prime \prime}(\xi)=f^{\prime}(\Phi) .
$$

Multiplying by $\Phi^{\prime}$, we get

$$
\frac{d}{d \xi} \frac{\Phi^{\prime 2}}{2}=\frac{d}{d \xi} f(\Phi(\xi))
$$

Copyright (c) by SIAM. Unauthorized reproduction of this article is prohibited. 
From the boundary conditions that $\Phi \rightarrow \pm 1$ and $\Phi^{\prime} \rightarrow 0$ as $\xi \rightarrow \pm \infty$, we have

$$
\frac{\Phi^{\prime 2}}{2}=f(\Phi(\xi))
$$

Since $f$ is a nonnegative function and $\Phi$ is a monotonic increasing function, we have $\Phi^{\prime}=(2 f(\Phi))^{1 / 2}$ and

$$
\sigma=\int_{-\infty}^{\infty}\left(\Phi^{\prime}\right)^{2} d \xi=\int_{-\infty}^{\infty}(2 f(\Phi))^{1 / 2} \Phi^{\prime} d \xi=\int_{-1}^{1}(2 f(\Phi))^{1 / 2} d \Phi=\tilde{\sigma}
$$

In addition, we could compute that

$$
\sigma=\tilde{\sigma}=\frac{1}{\sqrt{2}} \int_{-1}^{1}\left(1-r^{2}\right) d r=\frac{2 \sqrt{2}}{3} .
$$

Now we consider the value $\tilde{\gamma}(\mathbf{x}, \pm 1)$. We need the exact form of $\gamma(\mathbf{x}, s)$. From (3.28), we have

$$
\gamma(\mathbf{x}, s)=C-\frac{\sigma}{4} \cos \theta(\mathbf{x})\left(3 s-s^{3}\right)=C-\frac{\sqrt{2}}{6} \cos \theta(\mathbf{x})\left(3 s-s^{3}\right)
$$

for some constant $C$, which is independent of $s$. Then we could get, for $-1 \leq s, t \leq 1$,

$$
\begin{aligned}
F(\mathbf{x}, t, s) & =\gamma(\mathbf{x}, s)+\frac{\sqrt{2}}{2}\left|\int_{s}^{t}(2 f(r))^{1 / 2} d r\right| \\
& =C-\frac{\sqrt{2}}{6} \cos \theta(\mathbf{x})\left(3 s-s^{3}\right)+\frac{\sqrt{2}}{2}\left|\int_{s}^{t}\left(1-r^{2}\right) d r\right| .
\end{aligned}
$$

Direct computation shows that

$$
\tilde{\gamma}(\mathbf{x}, 1)=\min _{s} F(\mathbf{x}, 1, s)=F(\mathbf{x}, 1,1)=\gamma(\mathbf{x}, 1),
$$

and also

$$
\tilde{\gamma}(\mathbf{x},-1)=\min _{s} F(\mathbf{x},-1, s)=F(\mathbf{x},-1,-1)=\gamma(\mathbf{x},-1) .
$$

This completes the proof.

4.3. The convergence of the minimizers. Using Lemma 4.1 and Proposition 4.2 , we can prove the following theorem.

THEOREM 4.1. If $\phi_{0} \in B V(\Omega)$ is the isolated $L_{1}$-local minimizer of $I_{0}$, then there exists a sequence $\phi_{\epsilon_{j}} \in B V(\Omega)$ of the $L_{1}$-local minimizers of $I_{\epsilon_{j}}$ such that

$$
\lim _{j \rightarrow \infty}\left\|\phi_{\epsilon_{j}}-\phi_{0}\right\|_{L^{1}}=0
$$

as $\epsilon_{j} \rightarrow 0$.

Proof. We need only to show the lower semicontinuity and equicoercivity of the functional $I_{\epsilon}$ in the $L^{1}$ norm. The proof of the theorem is then standard.

First, we prove the lower semicontinuity. For some $\phi_{\infty} \in B V(\Omega)$, supposing a sequence $\phi_{j} \in H^{1}(\Omega)$ that converges to $\phi_{\infty}$ in $L^{1}(\Omega)$, we would like to show that

$$
I_{\epsilon}\left(\phi_{\infty}\right) \leq \liminf _{j \rightarrow \infty} I_{\epsilon}\left(\phi_{j}\right) .
$$

Copyright $@$ by SIAM. Unauthorized reproduction of this article is prohibited. 
Without loss of generality, we assume that there exists a constant $M_{1}$ such that

$$
\lim _{j \rightarrow \infty} I_{\epsilon}\left(\phi_{j}\right)=M_{1}<\infty .
$$

Since $f(\cdot) \geq 0$ and $\gamma(\cdot, \cdot) \geq 0$, then we could know that $\left|\phi_{j}\right|_{H^{1}(\Omega)}<M_{1} / \epsilon$. Thus, there exists a subsequence of $\phi_{j}$, without loss of generality, still denoted by $\phi_{j}$, such that

$$
\phi_{j} \rightarrow \phi_{\infty} \text { in } H^{1}(\Omega) \quad \text { and } \quad \phi_{j}(\mathbf{x}) \rightarrow \phi_{\infty}(\mathbf{x}) \text { a.e. } \mathbf{x} \in \Omega .
$$

Then, applying Fatou's lemma, (4.6) can be obtained by the weak lower semicontinuity of the functional $\epsilon \int_{\Omega}|\nabla \phi|^{2} d x$ (see [11]) and the continuity of $f(\cdot)$ and $\gamma(\mathbf{x}, \cdot)$.

Next, we show the equicoercivity of $I_{\epsilon}$. We need to prove that, for any sequences $\epsilon_{j} \rightarrow 0$ and $\phi_{j}$ such that $\sup _{j} I_{\epsilon_{j}}\left(\phi_{j}\right)<\infty$, there exists a subsequence of $\phi_{j}$ converging to some function $\phi$ in the $L^{1}$ norm. have

We suppose that there exists a constant $M_{2}$ such that $I_{\epsilon_{j}}\left(\phi_{j}\right)<M_{2}$; then we

$$
\int_{\Omega} \frac{\left(1-\phi_{j}^{2}\right)^{2}}{4} d x=\int_{\Omega} f\left(\phi_{j}\right) d x<M_{2} \epsilon_{j} \rightarrow 0 \quad \text { as } j \rightarrow \infty .
$$

This implies that there exists a subsequence of $\phi_{j}$, without loss of generality, still denoted by $\phi_{j}$, that converges in measure to some piecewise constant function $\phi$. It is also easy to see that $\phi_{j}$ are uniformly bounded in $L^{\infty}$, and hence from the convergence in measure, we have, up to a subsequence, that $\phi_{j}$ converge to the function $\phi$ in the $L^{1}$ norm.

4.4. The properties of the limiting minimizers. Now we study the geometric properties of the minimizers for the limiting functional $I_{0}$ in (4.2). The following theorem is a generalization of the results by Chen and Kowalczyk [6].

We assume that the patterned boundary $\partial \Omega=\mathcal{A} \cup \mathcal{B} \cup \mathcal{R}$, with $\mathcal{A}$ and $\mathcal{B}$ being two disjoint open subsets of $\partial \Omega$ and $\mathcal{R}=\left\{\mathbf{x}_{i}\right\}$ composed of some discrete points, and we suppose that the surface energy density function is defined as

$$
\gamma(\mathbf{x}, \phi)= \begin{cases}\gamma_{\mathcal{A}}(\phi), & \mathbf{x} \in \mathcal{A} \\ \gamma_{\mathcal{B}}(\phi), & \mathbf{x} \in \mathcal{B}\end{cases}
$$

Here $\gamma_{\mathcal{A}}(\phi)$ and $\gamma_{\mathcal{B}}(\phi)$ are such that

$$
\gamma_{\mathcal{A}}^{\prime}(\phi)=-\frac{\sigma}{2} \cos \theta_{\mathcal{A}} s_{\gamma}(\phi), \quad \gamma_{\mathcal{B}}^{\prime}(\phi)=-\frac{\sigma}{2} \cos \theta_{\mathcal{B}} s_{\gamma}(\phi),
$$

with $s_{\gamma}(\phi)=\frac{3}{2}\left(1-\phi^{2}\right)$. Without loss of generality, we assume that $0<\theta_{\mathcal{A}}<\theta_{\mathcal{B}}<\pi$.

THEOREM 4.2. Let $\phi_{0} \in B V(\Omega)$ be an $L_{1}$-local minimizer of $I_{0}$. Then we have

- $\phi_{0}(\mathbf{x})= \pm 1$, a.e. $\mathbf{x} \in \Omega$;

- the interface $\Gamma=\partial \Omega_{1} \cap \Omega$, with $\Omega_{1}=\left\{\mathbf{x} \in \Omega\right.$ : $\left.\phi_{0}(\mathbf{x})=1\right\}$, is of constant curvature;

- the contact angle of the phase $\left(\phi_{0}=1\right)$ at some contact point $\mathbf{x}_{0}$ is

$$
\theta_{s}=\left\{\begin{array}{cl}
\theta_{\mathcal{A}} & \text { if } \mathbf{x}_{0} \in \mathcal{A}, \\
\theta_{\mathcal{B}} & \text { if } \mathbf{x}_{0} \in \mathcal{B}, \\
\theta_{\zeta} & \text { if } \mathbf{x}_{0} \in \mathcal{R},
\end{array}\right.
$$

with some $\theta_{\zeta}$ such that $\theta_{\mathcal{A}} \leq \theta_{\zeta} \leq \theta_{\mathcal{B}}$. 
Proof. We first prove that the interface $\Gamma=\partial \Omega_{1} \cap \Omega$ is of constant curvature. For simplicity, we suppose that $\left|\Omega_{1}\right|$ is small and that $\Gamma$ is composed of a simple connected curve, which intersects with $\partial \Omega$ at two points $\mathbf{x}_{1}$ and $\mathbf{x}_{2}$.

Suppose that the curvature of $\Gamma$ is not constant. Then there exists a domain $\Omega_{2} \subset \Omega$ such that $\left|\Omega_{2}\right|=\left|\Omega_{1}\right|$, and $\tilde{\Gamma}=\partial \Omega_{2} \cap \Omega$ is a circular arc that intersects with $\partial \Omega$ at the two points $\mathbf{x}_{1}$ and $\mathbf{x}_{2}$. Then we extend $\Omega_{2}$ outside of $\Omega$ to a full circular disk $D$. From the geometric fact that a disk has the smallest circumference among all shapes with the same area, we have

$$
|\tilde{\Gamma}|<|\Gamma| .
$$

This implies that, for $\tilde{\phi}=\chi_{\Omega_{2}}-\chi_{\Omega \backslash \Omega_{2}}$, with $\chi_{\Omega_{2}}$ and $\chi_{\Omega \backslash \Omega_{2}}$ being the characteristic function of the domains $\Omega_{2}$ and $\Omega \backslash \Omega_{2}$, respectively,

$$
I_{0}(\tilde{\phi})<I_{0}(\phi) .
$$

One can define a homotopic mapping $H(s, \lambda), 0 \leq \lambda \leq 1$, with $H(s, 0)$ and $H(s, 1)$ representing the curves $\Gamma$ and $\tilde{\Gamma}$, respectively, such that the length of the curve $H(s, \lambda)$ is strictly monotonically decreasing with respect to $\lambda$ while the area $\Omega_{\lambda}$ bounded by the curve $H(s, \lambda)$ and $\partial \Omega$ keeps constant for any $\lambda$. Thus, the functions $\phi_{\lambda}=\chi_{\Omega_{\lambda}}-\chi_{\Omega \backslash \Omega_{\lambda}}$ converge to $\phi_{0}$ in the $L^{1}$ norm when $\lambda \rightarrow 0$, and

$$
I_{0}\left(\phi_{\lambda}\right)<I_{0}(\phi), \quad 0<\lambda<1 .
$$

This contradicts the fact that $\phi$ is an $L_{1}$-local minimizer of $I_{0}$. Thus, $\Gamma$ is of constant curvature.

Next, we study the contact angles of the phase $\phi_{0}(\mathbf{x})=1$ with respect to a local minimizer $\phi_{0}$. We follow the procedure used in [6]. We identify the points in $\Omega$ with complex numbers. Suppose that $z(\tau): \mathbb{R}^{1} \rightarrow \mathbb{C}$ is the counterclockwise oriented arclength parameterization of $\partial \Omega$. Then, $z^{\prime}(\tau)$ is the tangential direction of $\partial \Omega$ at the point $z(\tau)$. We then define the following complex function:

$$
w(t, \tau, \kappa, \theta)=z(\tau)+z^{\prime}(\tau) e^{i \theta} \int_{0}^{t} e^{i \kappa \tau} d \tau .
$$

It is easy to see that $w(\cdot, \tau, \kappa, \theta)$ is an arclength parameterization of circular arc that intersects $\partial \Omega$ at $z(\tau)$ with a contact angle $\theta$ and has curvature $\kappa$. Here the increasing direction of $w(t, \tau, \kappa, \theta)$ with respect to $t$ indicates the counterclockwise orientation. $\theta$ is defined as the angle of the counterclockwise rotation from $z^{\prime}(\tau)$ to $w_{t}(0, \tau, \kappa, \theta)$ (see Figure 4.1). Furthermore, one can show that (see [6]), for any positive constant $0<A<|\Omega|$, there exists a pair $(\tau, \varsigma)$, a positive curvature $\kappa$, an angle $\theta \in[0,2 \pi)$, and a constant $L \in(0,2 \pi /|\kappa|)$ such that

$$
\begin{aligned}
z(\varsigma) & =w(L, \tau, \kappa, \theta), \\
A & =\frac{1}{2} \operatorname{Im}\left\{\int_{0}^{L} \bar{w}(t, \tau, \kappa, \theta) w_{t}(t, \tau, \kappa, \theta) d t+\int_{\varsigma}^{\tau} \bar{z}(t) z^{\prime}(t) d t\right\} .
\end{aligned}
$$

This means that there exists a circular arc which intersects $\partial \Omega$ at $z(\tau)$ and $z(\varsigma)$, with the arclength $L$ and enclosed area $A$. Here $\theta, \kappa$, and $L$ are functions of $(\tau, \varsigma)$. Furthermore, we have

$$
\frac{\partial L}{\partial \tau}=-\cos \theta(\tau, \varsigma), \quad \frac{\partial L}{\partial \varsigma}=-\cos \vartheta(\tau, \varsigma),
$$






FIG. 4.1. The definition of $\theta$ and $\vartheta$.

where $\vartheta$ is the contact angle of the circular arc at $z(\varsigma)$, defined as the angle of the counterclockwise rotation from $\partial \Omega$ to the arc (see Figure 4.1).

Now we compute the derivative of $I_{0}\left(\phi_{0}\right)$ with respect to $\tau$ and $\varsigma$. For simplicity, we consider only the derivative in $\tau$. Suppose that the point $z(\tau)$ is in $\mathcal{A}$; then we have

$$
\begin{aligned}
\frac{\partial I_{0}}{\partial \tau} & =\sigma \frac{\partial L}{\partial \tau}+\frac{\partial}{\partial \tau}\left(\int_{\partial \Omega_{1} \cap \partial \Omega} \gamma(\mathbf{x}, 1) d s\right)+\frac{\partial}{\partial \tau}\left(\int_{\partial \Omega \backslash \partial \Omega_{1}} \gamma(\mathbf{x},-1) d s\right) \\
& =-\sigma \cos \theta+\gamma_{\mathcal{A}}(1)-\gamma_{\mathcal{A}}(-1) .
\end{aligned}
$$

Since $\phi_{0}$ is a local minimizer, we have $\frac{\partial I_{0}}{\partial \tau}=0$, which means

$$
-\sigma \cos \theta+\gamma_{\mathcal{A}}(1)-\gamma_{\mathcal{A}}(-1)=0 .
$$

Notice again that the contact angle of phase $\left(\phi_{0}=1\right)$ at the point $z(\tau)$ is $\theta_{s}=\pi-\theta$; thus we have

$$
\cos \theta_{s}=-\cos \theta=\frac{\gamma_{\mathcal{A}}(-1)-\gamma_{\mathcal{A}}(1)}{\sigma}=\cos \theta_{\mathcal{A}} .
$$

This implies that $\theta_{s}=\theta_{\mathcal{A}}$.

Similar analysis shows that, if the contact point is in $\mathcal{B}$, the local contact angle will be $\theta_{s}=\theta_{\mathcal{B}}$.

Now we consider the case when the contact point is in $\mathcal{R}$. In this case, the energy $I_{0}$ is not smooth in $\tau$, and we could compute only the left and right derivative of $I_{0}$. Suppose that the material on the left of $z(\tau)$ is $\mathcal{A}$, and the material on the right is $\mathcal{B}$. Then we could compute the left derivative as

$$
\begin{aligned}
\left.\frac{\partial I_{0}}{\partial \tau}\right|_{-} & =\sigma \frac{\partial L}{\partial \tau}+\left.\frac{\partial}{\partial \tau}\right|_{-}\left(\int_{\partial \Omega_{1} \cap \partial \Omega} \gamma(\mathbf{x}, 1) d s\right)+\left.\frac{\partial}{\partial \tau}\right|_{-}\left(\int_{\partial \Omega \backslash \partial \Omega_{1}} \gamma(\mathbf{x},-1) d s\right) \\
& =-\sigma \cos \theta+\gamma_{\mathcal{A}}(1)-\gamma_{\mathcal{A}}(-1) \\
& =\cos \theta_{s}-\cos \theta_{\mathcal{A}},
\end{aligned}
$$

and the right derivative as

$$
\begin{aligned}
\left.\frac{\partial I_{0}}{\partial \tau}\right|_{+} & =\sigma \frac{\partial L}{\partial \tau}+\left.\frac{\partial}{\partial \tau}\right|_{+}\left(\int_{\partial \Omega_{1} \cap \partial \Omega} \gamma(\mathbf{x}, 1) d s\right)+\left.\frac{\partial}{\partial \tau}\right|_{+}\left(\int_{\partial \Omega \backslash \partial \Omega_{1}} \gamma(\mathbf{x},-1) d s\right) \\
& =-\sigma \cos \theta+\gamma_{\mathcal{B}}(1)-\gamma_{\mathcal{B}}(-1) \\
& =\cos \theta_{s}-\cos \theta_{\mathcal{B}} .
\end{aligned}
$$

Copyright $@$ by SIAM. Unauthorized reproduction of this article is prohibited. 
Since $I_{0}$ is a minimizer, we must have

$$
\left.\frac{\partial I_{0}}{\partial \varsigma}\right|_{-} \leq 0,\left.\quad \frac{\partial I_{0}}{\partial \varsigma}\right|_{+} \geq 0
$$

This implies the following condition for the contact angles:

$$
\theta_{\mathcal{A}} \leq \theta_{s} \leq \theta_{\mathcal{B}}
$$

The situation for $z(\varsigma)$ can be proved in a similar way. This completes our proof.

Remark 4.2. In the proof of the theorem, we did not consider the situation when the material on the left of $z(\tau)$ is $\mathcal{B}$ and the material on the right is $\mathcal{A}$. In fact, for a $\phi$ corresponding to such a configuration, we always have

$$
\left.\frac{\partial I_{0}(\phi)}{\partial \tau}\right|_{-}=\cos \theta_{s}-\cos \theta_{\mathcal{B}}>\cos \theta_{s}-\cos \theta_{\mathcal{A}}=\left.\frac{\partial I_{0}(\phi)}{\partial \tau}\right|_{+} .
$$

This means that the function $\phi$ cannot be a local minimizer in this case. As we will see in the examples in next section, the contact line jumps at such a point.

5. Some examples for wetting hysteresis. The results in the previous sections provide a clear geometric picture of the equilibrium configuration in two dimensions. This enables us to explicitly calculate and minimize the total surface energy for some two phase fluid systems with simple geometry. By considering the quasistatic motion of the interface on the patterned surfaces, described by the change of volume (or volume fraction), we can follow the change of the energy landscape, the change of contact angles, and CAH. In this section, we study two explicit examples: the Poiseuille-type flow on a patterned surface and a drop spreading on a patterned surface.



FIG. 5.1. The channel consists of two materials.

5.1. Quasi-static flow in a chemically patterned channel. We first consider a two phase quasi-static flow in a channel, as shown in Figure 5.1. The height of the channel is $2 h$. The length of the channel is $2 L$, with the lower side denoted by $A C$ and the middle point denoted by $B$. In the following examples, we always choose $h=4, L=10$. The channel is patterned with two materials with equal length $A B$ and $B C$ with contact angles $\theta_{\mathcal{A}}$ and $\theta_{\mathcal{B}}$, respectively. The fluid interface energy density is given by $\gamma$. Denote the domain occupied by fluid 1 as $\Omega_{1}$, and the domain occupied by fluid 2 as $\Omega_{2}$. The results from the previous sections show that the interface is part of a circle with a contact point $D$ on the lower side and a contact angle $\theta$ of fluid 2 on the solid surface. The length of the interval $A D$ is denoted by $x$. The difference of the areas are measured by a parameter $\alpha$ defined as follows:

$$
\left|\Omega_{1}\right|-\left|\Omega_{2}\right|=\alpha \cdot(2 L) \cdot(2 h)
$$


so that $-1<\alpha<1$. From (5.1), it is easy to show that $x$ is related to $\theta$ via

$$
x=L-\frac{1}{2} \frac{h}{\cos ^{2} \theta}\left(\left(\frac{\pi}{2}-\theta\right)-\cos \theta \sin \theta\right)+\alpha L
$$

or, equivalently,

$$
\hat{x}=\frac{x-L}{h}=-\frac{1}{2 \cos ^{2} \theta}\left(\frac{\pi}{2}-\theta-\cos \theta \sin \theta\right)+\alpha \frac{L}{h} .
$$

Notice that for any fixed $\alpha, \hat{x}$ is a monotone-increasing function of $\theta$.

We denote by $\Sigma_{0}, \Sigma_{1}$, and $\Sigma_{2}$ the fluid-fluid interface, solid-fluid1 interface, and solid-fluid2 interface, respectively. Notice that $\cos \theta_{\mathcal{A}}=\frac{\gamma_{1, \mathcal{A}}-\gamma_{2, \mathcal{A}}}{\gamma}$ and $\cos \theta_{\mathcal{B}}=$ $\frac{\gamma_{1, \mathcal{B}}-\gamma_{2, \mathcal{B}}}{\gamma}$. Then, the normalized total surface energy of the system can be calculated in terms of $\theta$ and $\alpha$ :

$$
\hat{E}(\theta, \alpha)=\frac{E}{\gamma h}=\frac{\gamma\left|\Sigma_{0}\right|+\gamma_{1}\left|\Sigma_{1}\right|+\gamma_{2}\left|\Sigma_{2}\right|}{\gamma h}=\frac{(\pi-2 \theta)}{\cos \theta}+2 \hat{x} \beta+e_{0},
$$

where $e_{0}$ is a constant independent of $\theta$ and $\alpha, \hat{x}$ is given by (5.2), and

$$
\beta= \begin{cases}\cos \theta_{\mathcal{A}} & \text { if } \hat{x}<0 \\ \cos \theta_{\mathcal{B}} & \text { if } \hat{x}>0\end{cases}
$$

To understand the stick-slip motion of the interface and the CAH, we consider quasi-static motion of the interface as $\alpha$ changes. The increasing or decreasing of parameter $\alpha$ then corresponds to the right or left motion of the interface. For each fixed $\alpha$, the stable configurations can be obtained from locally minimizing the normalized total surface energy (5.3) with respect to $\theta$. The corresponding contact point $\hat{x}$ and contact angle $\theta_{s}$ are also obtained. We separately study the stick $\left(\theta_{\mathcal{A}}>\theta_{\mathcal{B}}\right)$ and $\operatorname{slip}\left(\theta_{\mathcal{A}}<\theta_{\mathcal{B}}\right)$ cases.

Contact line slip. We first consider the case when $\theta_{\mathcal{A}}<\theta_{\mathcal{B}}$. In the upper row of Figure 5.2, we plot $\hat{E}(\theta, \alpha)$ as a function of $\theta$ for different values of $\alpha$. It is easy to show that there are two critical $\alpha$ values,

$$
\alpha_{-}=\frac{h}{2 L}\left(\frac{\pi / 2-\theta_{\mathcal{B}}}{\cos ^{2}\left(\theta_{\mathcal{B}}\right)}-\tan \left(\theta_{\mathcal{B}}\right)\right), \quad \alpha_{+}=\frac{h}{2 L}\left(\frac{\pi / 2-\theta_{\mathcal{A}}}{\cos ^{2}\left(\theta_{\mathcal{A}}\right)}-\tan \left(\theta_{\mathcal{A}}\right)\right),
$$

such that the normalized energy $\hat{E}(\theta, \alpha)$ experiences a transition from a single well function (in $\theta$ ) to a double well function (or vice versa) at $\alpha_{-}$and $\alpha_{+}$. For $\alpha<\alpha_{-}$, $\hat{E}$ has a unique minimum at $\theta_{\mathcal{A}}$. For $\alpha_{-}<\alpha<\alpha_{+}, \hat{E}$ has two local minima at $\theta_{\mathcal{A}}$ and $\theta_{\mathcal{B}}$. For $\alpha>\alpha_{+}, \hat{E}$ has a unique minimum at $\theta_{\mathcal{B}}$. The existence of multiple local minima for $\hat{E}$ for $\alpha_{-}<\alpha<\alpha_{+}$(i.e., when the interface is close to the transition point of the pattern) is the origin for the CAH. In the lower row of Figure 5.2, we plot the stable contact angle $\theta_{s}$ and the contact point $\hat{x}$ as the functions of $\alpha$. As $\alpha$ increases (or interface moves from left to right) and passes $\alpha_{-}$, the system chooses to stay in the same local minimum at the contact angle $\theta_{\mathcal{A}}$, since it costs energy to switch to the other minimum. When $\alpha$ passes $\alpha_{+}, \theta_{\mathcal{A}}$ is no longer a local minimum, and the contact angle has to switch instantly to $\theta_{\mathcal{B}}$ (which is now the only local minimum) as the contact point jumps a distance $\Delta l$, where

$$
\Delta l=\frac{h}{2}\left|\left(\frac{\pi / 2-\theta_{\mathcal{A}}}{\cos ^{2} \theta_{\mathcal{A}}}-\frac{\pi / 2-\theta_{\mathcal{B}}}{\cos ^{2} \theta_{\mathcal{B}}}\right)+\left(\tan \theta_{\mathcal{B}}-\tan \theta_{\mathcal{A}}\right)\right| .
$$

Copyright $@$ by SIAM. Unauthorized reproduction of this article is prohibited. 

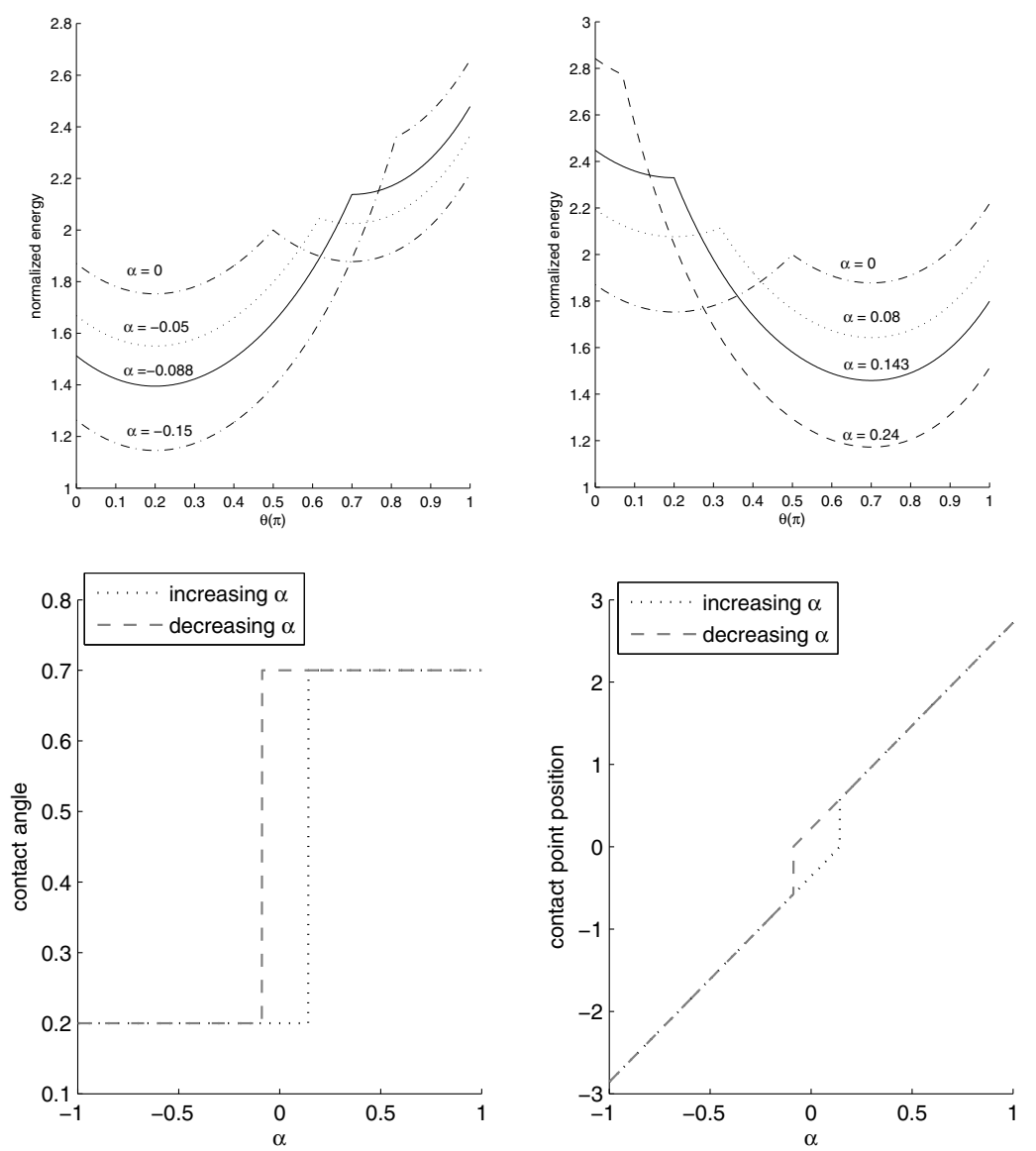

FIG. 5.2. Contact angle hysteresis and contact line slipping. Upper row: The normalized energy (5.3) as a function of $\theta$ for different $\alpha$. The solid lines are $\hat{E}(\theta)$ at $\alpha_{-}$and $\alpha_{+_{+}}$. Lower row: Contact angle (left) and contact point (right) as functions of $\alpha$. Here $\theta_{\mathcal{A}}=\frac{\pi}{5}, \theta_{\mathcal{B}}=\frac{7 \pi}{10}, \alpha_{-}=-0.088$, and $\alpha_{+}=0.143$.

On the other hand, when the interface moves from right to left, the stable contact angle chooses to stay at $\theta_{\mathcal{B}}$ before it has to switch to $\theta_{\mathcal{A}}$ at $\alpha=\alpha_{-}$. We therefore observe hysteresis in both cases.

Contact line pinning. The case when $\theta_{\mathcal{A}}>\theta_{\mathcal{B}}$ corresponds to the pinning (stick) of the interface. In Figure 5.3, we plot the energy $\hat{E}(\theta, \alpha)$ for different values of $\alpha$ in the upper row and for stable contact angle $\theta_{s}$ and contact point $\hat{x}$ in the lower row. It is easy to see that $\hat{E}(\theta, \alpha)$ always has a unique minimum at $\theta_{s}$ for all $\alpha$ and

$$
\theta_{s}= \begin{cases}\theta_{\mathcal{A}} & \text { if } \alpha \leq \alpha_{-} \\ \theta_{s} \text { determined by (5.2) with } \hat{x}=0 & \text { if } \alpha \alpha_{-}<\alpha_{+}, \\ \theta_{\mathcal{B}} & \text { if } \alpha \geq \alpha_{+}\end{cases}
$$

Here $\alpha_{-}$and $\alpha_{+}$are now given by

$$
\alpha_{-}=\frac{h}{2 L}\left(\frac{\pi / 2-\theta_{\mathcal{A}}}{\cos ^{2}\left(\theta_{\mathcal{A}}\right)}-\tan \left(\theta_{\mathcal{A}}\right)\right), \quad \alpha_{+}=\frac{h}{2 L}\left(\frac{\pi / 2-\theta_{\mathcal{B}}}{\cos ^{2}\left(\theta_{\mathcal{B}}\right)}-\tan \left(\theta_{\mathcal{B}}\right)\right) .
$$

Copyright $@$ by SIAM. Unauthorized reproduction of this article is prohibited. 

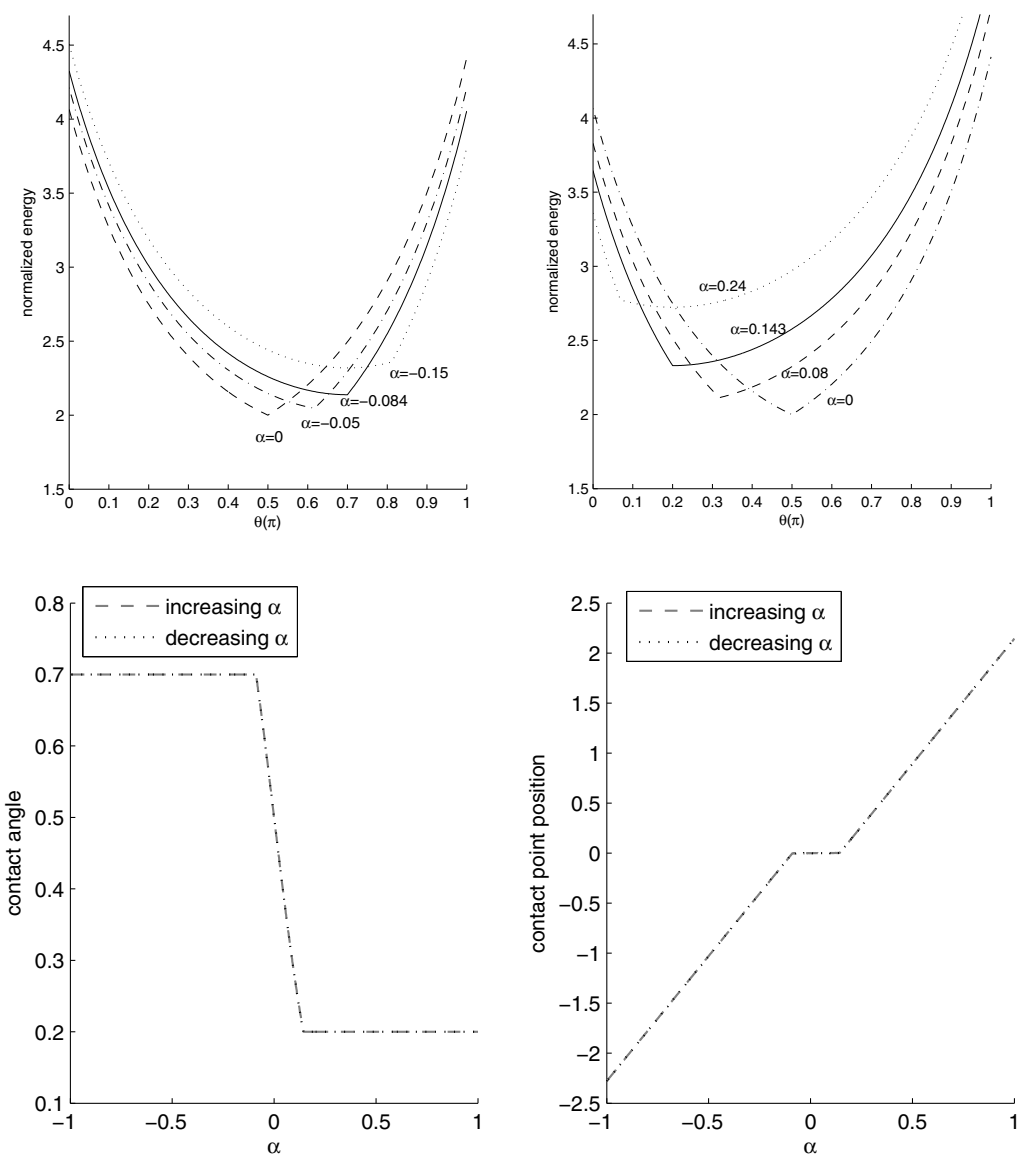

FIG. 5.3. Contact line pinning. Upper row: The normalized energy as a function of $\theta,(5.3)$, for different $\alpha$. Lower row: Contact angle (left) and contact point (right) as functions of $\alpha$. Here $\theta_{\mathcal{A}}=\frac{7 \pi}{10}, \theta_{\mathcal{B}}=\frac{\pi}{5}, \alpha_{-}=-0.088$, and $\alpha_{+}=0.143$.

As $\alpha$ increases (or as the interface moves from left to right) and reaches $\alpha_{-}$, the interface is pinned at point $B(\hat{x}=0)$ as $\alpha$ continues to increase from $\alpha_{-}$to $\alpha_{+}$, and the contact angle switches gradually from $\theta_{\mathcal{A}}$ to $\theta_{\mathcal{B}}$. As $\alpha$ decreases (or the interface goes back from right to left), it follows exactly the same path. The contact point is pinned at the same position, and the contact angle switches gradually from $\theta_{\mathcal{B}}$ to $\theta_{\mathcal{A}}$. No hysteresis is observed in this case.

We next consider a channel periodically patterned with materials with different contact angles $\theta_{\mathcal{A}}$ and $\theta_{\mathcal{B}}$ on the solid boundary (Figure 5.4). We assume that there are $k$ periodic patterns in the interval $\left[\frac{L}{2}, \frac{L}{2}\right]$. In each period, the two materials occupy the same area $\Delta x=\frac{L}{2 k}$. The normalized energy can be computed as follows:

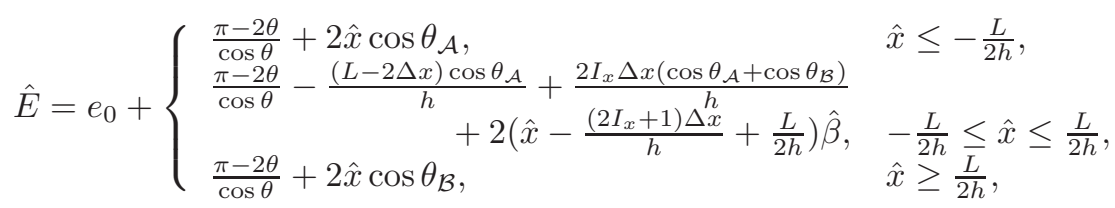

Copyright (c) by SIAM. Unauthorized reproduction of this article is prohibited. 


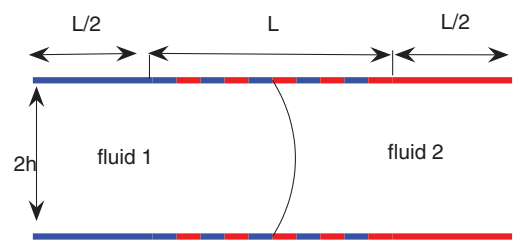

FIG. 5.4. The chemically patterned channels.


FIG. 5.5. The contact angle and contact point as functions of $\alpha$ with $k=2$. A simple repeat of the stick-slip motion and contact angle switching and $C A H . \theta_{\mathcal{A}}=\frac{\pi}{5}, \theta_{\mathcal{B}}=\frac{7 \pi}{10}$.

where $I_{x}=\left[\frac{2 \hat{x} h+L}{4 \Delta x}\right]$ is the integer part of the number $\frac{2 \hat{x} h+L}{4 \Delta x}=\frac{x-L / 2}{2 \Delta x}$, representing the number of complete periods occupied by fluid 1 , and

$$
\hat{\beta}= \begin{cases}\cos \theta_{\mathcal{A}} & \text { if } \frac{2 \hat{x} h+L}{4 \Delta x}-I_{x} \leq \frac{1}{2} \\ \cos \theta_{\mathcal{B}} & \text { otherwise. }\end{cases}
$$

In the examples below, we take $\theta_{\mathcal{A}}=\frac{\pi}{5}$ and $\theta_{\mathcal{B}}=\frac{7 \pi}{10}$.

We now study the behavior of the quasi-static motion of the interface as the period $k$ increases (or as the size of the pattern $\Delta x$ decreases). Three different cases are shown in Figures $5.5(k=2), 5.6(k=5)$, and $5.7(k=15)$, where the contact angle $\theta_{s}$ and the contact point $\hat{x}$ are plotted as a function of $\alpha$. For $k=2$, the behavior of the interface is a simple repeat of the stick-slip behavior described in Figure 5.3. For $k=5,15$, we see that the amplitude of the stick-slip becomes weaker as the size of the pattern $\Delta x$ decreases. When $\Delta x<\Delta l$, the contact angle cannot switch completely between $\theta_{\mathcal{A}}$ and $\theta_{\mathcal{B}}$ because there is no room for such a switching. In fact, as $\Delta x$ becomes smaller and smaller, the contact angle oscillates around $\theta_{\mathcal{B}}$ (or $\theta_{\mathcal{A}}$ ) as the interface moves to the left (or to the right), displaying apparent advancing (or receding) contact angle (Figures 5.6 and 5.7).

5.2. A drop spreading on a patterned surface. We now study the hysteresis behavior of a drop spreading on a patterned surface. We assume that the surface is periodically patterned in the interval $(-R, R)$, as shown in Figure 5.8. We assume that the interval $(-R, R)$ is divided into $k+\frac{1}{2}$ periods with equal partition of two materials. The half period in the center is occupied by the material $\mathcal{B}$. Let $\Delta x=\frac{2 R}{4 k+1}$ 

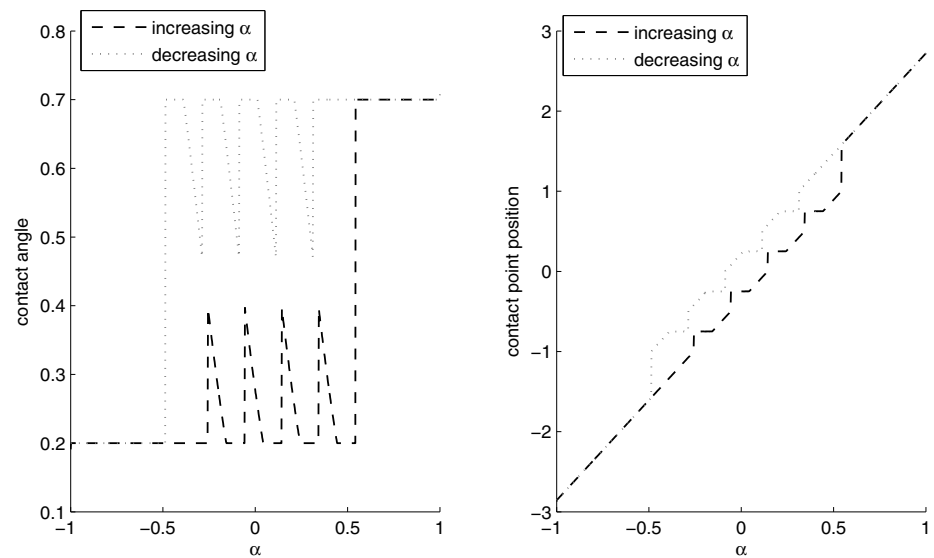

FIG. 5.6. The contact angle and contact point as functions of $\alpha$ with $k=5$. Here the pattern size $\Delta x<\Delta l$, so that contact angle will not switch. $\theta_{\mathcal{A}}=\frac{\pi}{5}, \theta_{\mathcal{B}}=\frac{7 \pi}{10}$.
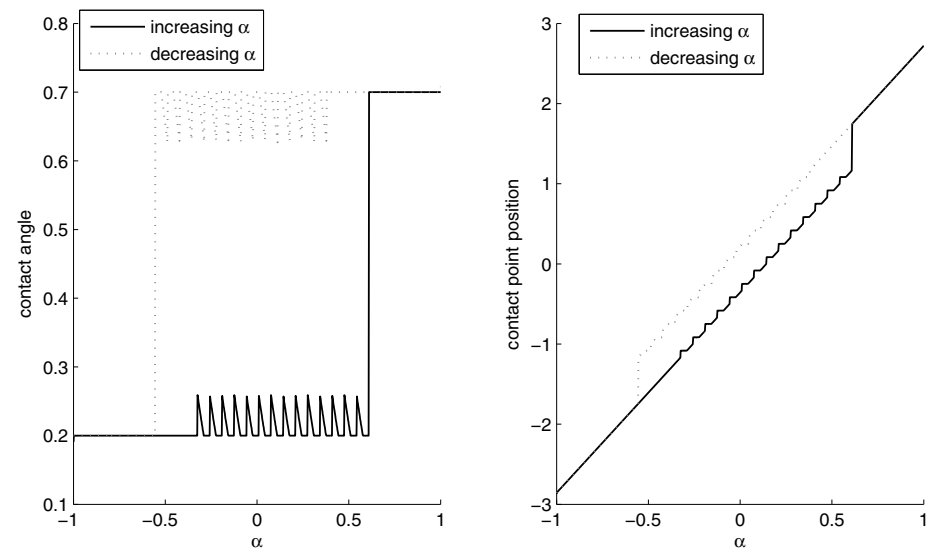

FIG. 5.7. The contact angle and contact point as functions of $\alpha$ with $k=15$. Here the pattern size is small enough so that one observes clear advancing and receding contact angles. $\theta_{\mathcal{A}}=\frac{\pi}{5}$, $\theta_{\mathcal{B}}=\frac{7 \pi}{10}$.



(a) Advancing angle.

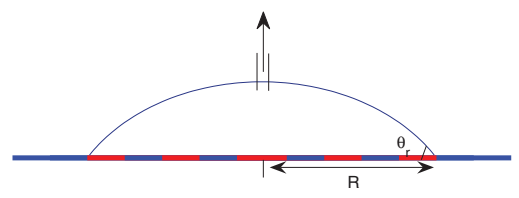

(b) Receding angle.

FIG. 5.8. Drop spreading and receding on a patterned surface.

Copyright (c) by SIAM. Unauthorized reproduction of this article is prohibited. 

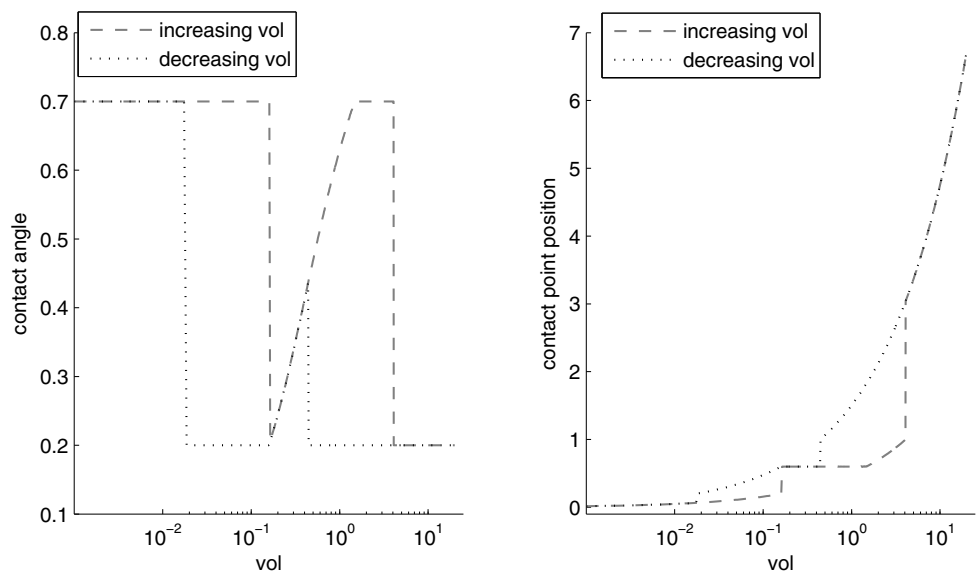

FIG. 5.9. The $C A H$ and stick-slip motion of a drop with $k=1$. $\theta_{\mathcal{A}}=\frac{\pi}{5}, \theta_{\mathcal{B}}=\frac{7 \pi}{10}$.

denote the length of a half period. Let $r$ be the radius of the drop on the solid surface and $\theta$ be the contact angle. Then for any fixed drop volume $V, r$ will be a function of the contact angle $\theta$, and the total surface energy $E$ will also be a function of $r(\theta)$ and $\theta$. We then compute the normalized radius $\hat{r}$,

$$
\hat{r}=\frac{r}{R}=\frac{\sqrt{V} \sin \theta}{R \sqrt{\theta-\sin \theta \cos \theta}} .
$$

The total normalized energy can be computed as

$$
\begin{aligned}
& \hat{E}(\theta, V)=\frac{E}{2 \gamma R} \\
& (5.7)=e_{1}+\frac{\hat{r} \theta}{\sin \theta}+ \begin{cases}-\hat{r} \cos \theta_{\mathcal{B}}, & \hat{r}<\frac{\Delta x}{2 R}, \\
-\left(\hat{r}-\frac{\left(4 I_{r}+3\right) \Delta x}{2 R}\right) \beta-\frac{\left(\cos \theta_{\mathcal{A}}+\cos \theta_{\mathcal{B}}\right)\left(1+2 I_{r}\right) \Delta x}{2 R} & \frac{\Delta x \cos \theta_{\mathcal{A}}{ }^{2 R},}{2 R} \leq \hat{r} \leq 1, \\
-\hat{r} \cos \theta_{\mathcal{A}}+\frac{2 \hat{r}+\Delta x / R}{4}\left(\cos \theta_{\mathcal{A}}-\cos \theta_{\mathcal{B}}\right), & \hat{r} \geq 1,\end{cases}
\end{aligned}
$$

where $e_{1}$ is a constant independent of $V$ and $\theta, I_{r}=\left[\frac{2 r-\Delta x}{4 \Delta x}\right]$ is the integer part of the number $\frac{r-\Delta x / 2}{2 \Delta x}$ representing the number of complete periods contained in the distance $r$, and

$$
\beta= \begin{cases}\cos \theta_{\mathcal{A}} & \text { if } \frac{2 r-\Delta x}{4 \Delta x}-I_{r} \leq \frac{1}{2} \\ \cos \theta_{\mathcal{B}} & \text { otherwise. }\end{cases}
$$

In Figures $5.9(k=1)$ and $5.10(k=15)$, we plot the contact angle $\theta_{s}$ and the contact point $\hat{x}$ as functions of the volume $V$ (in $\log$ scale). In both cases, we take $\theta_{\mathcal{A}}=\frac{\pi}{5}$ and $\theta_{\mathcal{B}}=\frac{7 \pi}{10}$. The phenomena here are similar to those described in the previous section for the channel flow. For $k=1$, the contact point goes through stickslip motion, and the contact angle switches between $\theta_{\mathcal{A}}$ and $\theta_{\mathcal{B}}$ displaying hysteresis. When the scale of the pattern is small (for $k=15$ ), we observe weaker stick-slip but stronger hysteresis, displaying the advancing contact angle $\theta_{\mathcal{B}}$ when the volume of the drop is increasing, and the receding contact angle $\theta_{\mathcal{A}}$ when the volume of the drop 

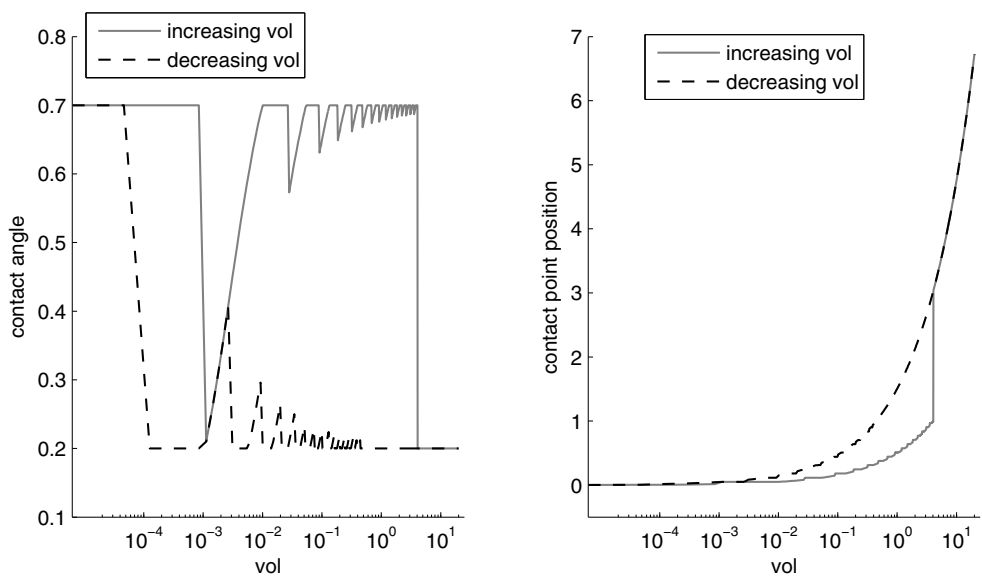

FIG. 5.10. The $C A H$ displaying advancing and receding contact angles. Here $k=15 . \theta_{\mathcal{A}}=\frac{\pi}{5}$, $\theta_{\mathcal{B}}=\frac{7 \pi}{10}$.

is decreasing. However, in the drop spreading case, the amount of the contact point jump $\Delta l$ (when experiencing a complete switching between $\theta_{\mathcal{A}}$ and $\theta_{\mathcal{B}}$ ) is volume dependent and is given by

$$
\Delta l=\sqrt{V}\left|\frac{\sin \theta_{\mathcal{B}}}{\sqrt{\theta_{\mathcal{B}}-\sin \theta_{\mathcal{B}} \cos \theta_{\mathcal{B}}}}-\frac{\sin \theta_{\mathcal{A}}}{\sqrt{\theta_{\mathcal{A}}-\sin \theta_{\mathcal{A}} \cos \theta_{\mathcal{A}}}}\right| .
$$

Therefore the oscillations near the advancing and receding angles not only decrease with size of the pattern $\Delta x$ but also decrease with volume of the drop $V$.

\section{Appendix.}

Proof of Proposition 4.1. We need only to prove the following two lemmas for the lower-bound and upper-bound inequalities of $\Gamma$-convergence $[3,7]$.

Lemma A.1 (the lower-bound inequality). For any $\phi_{\epsilon} \in B V(\Omega)$, if $\phi_{\epsilon} \rightarrow \phi_{0}$ in $L^{1}(\Omega)$ and $\liminf _{\epsilon \rightarrow 0} I_{\epsilon}\left(\phi_{\epsilon}\right)<\infty$, then we have $\phi_{0} \in B V(\Omega), \phi_{0}= \pm 1$, a.e., $\int_{\Omega} \phi=C_{0}$ and

$$
\tilde{I}_{0}\left(\phi_{0}\right) \leq \liminf _{\epsilon \rightarrow 0} I_{\epsilon}\left(\phi_{\epsilon}\right) .
$$

Proof. Since $\phi_{\epsilon} \rightarrow \phi_{0}$ in $L^{1}$, we can choose a subsequence (still denoted by $\phi_{\epsilon}$ ) such that $\phi_{\epsilon} \rightarrow \phi$ a.e. $x \in \Omega$. So $f\left(\phi_{\epsilon}\right) \rightarrow f(\phi)$ a.e. $x \in \Omega$. By Fatou's lemma, we have

$$
\int_{\Omega} f\left(\phi_{0}\right) d x \leq \liminf _{\epsilon \rightarrow 0} \int_{\Omega} f\left(\phi_{\epsilon}\right) \leq \liminf _{\epsilon \rightarrow 0} \epsilon I\left(\phi_{\epsilon}\right)=0 .
$$

Notice that $f\left(\phi_{0}\right) \geq 0$, and we have $f\left(\phi_{0}(x)\right)=0$ a.e. $x \in \Omega$. This implies that $\phi_{0}= \pm 1, x \in \Omega$.

Notice that $I_{\epsilon}\left(\phi_{\epsilon}\right)<\infty$. From the definition of the $I_{\epsilon}$, we know that $\int_{\Omega} \phi_{\epsilon}=C_{0}$. Notice again $\phi_{\epsilon} \rightarrow \phi_{0}$ in $L^{1}(\Omega)$; we have $\int_{\Omega} \phi_{0}=C_{0}$.

To prove the lower-bound inequality (A.1), we define the function

$$
F(t)=\int_{-1}^{t}(2 f(r))^{\frac{1}{2}} d r .
$$

Copyright $@$ by SIAM. Unauthorized reproduction of this article is prohibited. 
We then have

$$
\begin{aligned}
\int_{\Omega}\left|D F\left(\phi_{\epsilon}\right)\right| d x & \leq \int_{\Omega}\left|F^{\prime}\left(\phi_{\epsilon}\right)\right|\left|\nabla \phi_{\epsilon}\right| d x \\
& =\int_{\Omega}\left(2 f\left(\phi_{\epsilon}\right)\right)^{\frac{1}{2}}\left|\nabla \phi_{\epsilon}\right| d x \\
& \leq \int_{\Omega} \epsilon f\left(\phi_{\epsilon}\right)+\frac{\left|\nabla \phi_{\epsilon}\right|^{2}}{2 \epsilon} d x .
\end{aligned}
$$

Using the inequality $\tilde{\gamma}(x, \phi) \leq \gamma(x, \phi)$, we have

$$
\int_{\Omega}\left|D F\left(\phi_{\epsilon}\right)\right| d x+\int_{\partial \Omega} \tilde{\gamma}\left(x, \phi_{\epsilon}\right) d s \leq I_{\epsilon}\left(\phi_{\epsilon}\right)<\infty .
$$

In addition, similar to Proposition 1.4 in [15], we can show that $F\left(\phi_{0}\right) \in B V(\Omega)$ and

$$
\begin{aligned}
\int_{\Omega}\left|D F\left(\phi_{0}\right)\right| d x+\int_{\partial \Omega} \tilde{\gamma}\left(x, \phi_{0}\right) d s & \leq \liminf _{\epsilon \rightarrow 0} \int_{\Omega}\left|D F\left(\phi_{\epsilon}\right)\right| d x+\int_{\partial \Omega} \tilde{\gamma}\left(x, \phi_{\epsilon}\right) d s \\
& \leq \liminf _{\epsilon \rightarrow 0} I_{\epsilon}\left(\phi_{\epsilon}\right) .
\end{aligned}
$$

Notice also that

$$
\int_{\Omega}\left|D F\left(\phi_{0}\right)\right| d x=\int_{-\infty}^{+\infty} P_{\Omega}\left(\left\{x \in \Omega: F\left(\phi_{0}(x)\right)>t\right\}\right) d t=\int_{F(-1)}^{F(1)} P_{\Omega_{1}} d t=\tilde{\sigma}\left|\partial \Omega_{1} \cap \Omega\right|,
$$

with $P_{\Omega}(\tilde{\Omega})=|\partial \tilde{\Omega} \cap \Omega|$ and $\Omega_{1}=\left\{x \in \Omega: \phi_{0}(x)=1\right\}$. We have $\phi_{0} \in B V(\Omega)$ and $\tilde{I}_{0}\left(\Omega_{0}\right) \leq \liminf _{\epsilon \rightarrow 0} I_{\epsilon}\left(\phi_{\epsilon}\right)$.

Remark A.1. The original version of Proposition 1.4 in [15] is for the homogeneous boundary condition. This is

$$
\int_{\Omega}\left|D F\left(\phi_{0}\right)\right| d x+\int_{\partial \Omega} \tilde{\gamma}\left(\phi_{0}\right) d s \leq \liminf _{\epsilon \rightarrow 0} \int_{\Omega}\left|D F\left(\phi_{\epsilon}\right)\right| d x+\int_{\partial \Omega} \tilde{\gamma}(\phi) d s .
$$

However, this result is also true for the inhomogeneous boundary condition $\tilde{\gamma}(x, \phi)$, because the proof of Proposition 1.4 in [15] is based on Proposition 1.2 in the same paper, which is originally for the general boundary condition $\tilde{\gamma}(x, \phi)$.

Lemma A.2 (the upper-bound inequality). For any $\phi_{0} \in B V(\Omega), \phi_{0}= \pm 1$, a.e. $x \in \Omega, \int_{\Omega} \phi_{0} d x=C_{0}$, such that $I_{0}\left(\phi_{0}\right)<\infty$, we have

$$
\limsup _{\epsilon \rightarrow 0^{+}} \inf _{\phi \in U_{r}\left(\phi_{0}\right)} I_{\epsilon}(\phi) \leq I_{0}\left(\phi_{0}\right),
$$

with

$$
U_{r}\left(\phi_{0}\right)=\left\{\phi \in H^{1}(\Omega):\left\|\phi-\phi_{0}\right\|_{L^{2}(\Omega)}<r, \int_{\Omega} \phi d x=\int_{\Omega} \phi_{0} d x\right\} .
$$

Proof. For any $\phi_{0} \in B V(\Omega), \phi_{0}= \pm 1$, a.e. $x \in \Omega, \int_{\Omega} \phi_{0} d x=C_{0}$, we set $\Omega_{1}=\{x \in \Omega: \phi(x)=1\}$. As in [15], we could construct a sequence $\tilde{\phi}_{\epsilon}$, which is also shown in Figure A.1. Let $\Gamma=\partial \Omega_{1} \cap \Omega, \Omega_{2}=\Omega \backslash \Omega_{1}$. The domain $\Omega$ is divided into several parts:

$$
\begin{aligned}
& A_{1}=\Omega_{1} \cap\left\{x \in \Omega, d(x, \partial \Omega)>C_{1} \epsilon\right\}, \\
& A_{2}=\left(\Omega_{2} \cap\left\{x \in \Omega, d(x, \partial \Omega)>C_{1} \epsilon\right\} \cap\left\{x \in \Omega, d(x, \Gamma)>C_{1} \epsilon\right\}\right) \backslash B_{\epsilon},
\end{aligned}
$$

Copyright (c) by SIAM. Unauthorized reproduction of this article is prohibited. 


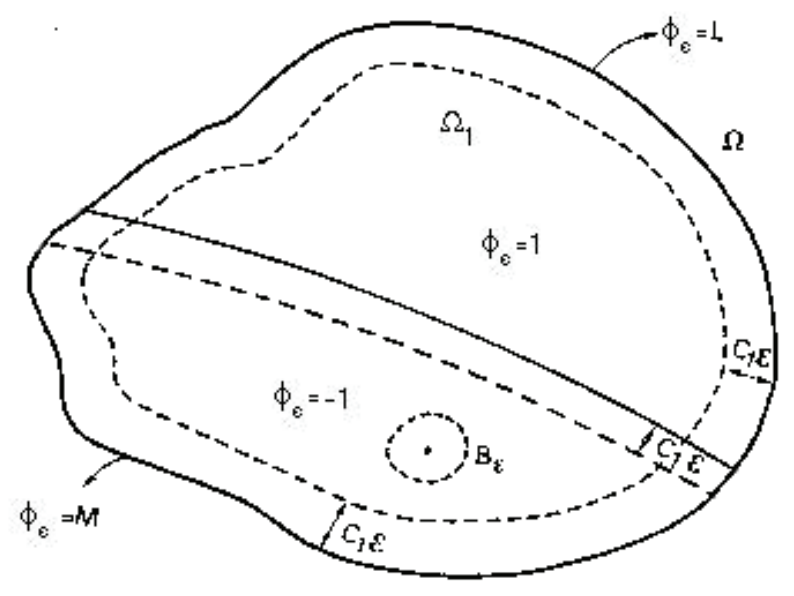

FIG. A.1. The construction of $\phi_{\epsilon}$ in [15].

with $B_{\epsilon}=\left\{x \in \Omega_{2}:\left|x-x_{0}\right|<\epsilon^{1 / 2}\right\}$ for some $x_{0} \in \Omega_{2}, d\left(x_{0}, \partial \Omega\right)>\epsilon^{\frac{1}{2}}$, and other boundary layers.

The $\phi_{\epsilon}$ is defined as

$$
\phi_{\epsilon}(x)= \begin{cases}1, & x \in A_{1}, \\ -1, & x \in A_{2}, \\ L, & x \in \partial \Omega_{1} \cap \partial \Omega, \\ M, & x \in \partial \Omega_{2} \cap \partial \Omega \cap\left\{x \in \partial \Omega: d(x, \Gamma)>C_{1} \epsilon\right\} .\end{cases}
$$

$\phi_{\epsilon}$ is set to some functions continuous to the boundary in the boundary layers and in $B_{\epsilon}$. For simplicity, we do not give the exact definition of these continuous functions; readers who are interested are referred to the definitions in [15]. The constructed functions $\phi_{\epsilon}$ are such that the following conditions hold (see in [15]):

$$
\lim _{\epsilon \rightarrow 0} \int_{\Omega}\left|\phi_{\epsilon}-\phi_{0}\right|^{2} d x=1, \quad \int_{\Omega} \phi_{\epsilon} d x=C_{0}
$$

and

$$
\begin{aligned}
\limsup _{\epsilon \rightarrow 0} \int_{\Omega} \epsilon\left|\nabla \phi_{\epsilon}\right|^{2}+\frac{2}{\epsilon} f\left(\phi_{\epsilon}\right) d x \leq & 2\left|\partial \Omega_{1} \cap \Omega\right| \int_{-1}^{1}(\delta+2 f(t))^{1 / 2} d t \\
& +2\left|\partial \Omega_{2} \cap \partial \Omega\right|\left|\int_{-1}^{M}(\delta+2 f(t))^{1 / 2} d t\right| \\
& +2\left|\partial \Omega_{1} \cap \partial \Omega\right|\left|\int_{1}^{L}(\delta+2 f(t))^{1 / 2} d t\right| .
\end{aligned}
$$

Here $\delta>0$ is a number independent of $L$ and $M$. 
Thus, we have, for any $r>0$,

$$
\begin{aligned}
\limsup _{\epsilon \rightarrow 0} \inf _{\phi \in U_{r}\left(\phi_{0}\right)} I_{\epsilon}(\phi) \leq & \limsup _{\epsilon \rightarrow 0} I_{\epsilon}\left(\phi_{\epsilon}\right) \\
= & \limsup _{\epsilon \rightarrow 0} \int_{\Omega} \frac{\epsilon}{2}\left|\nabla \phi_{\epsilon}\right|^{2}+\frac{f\left(\phi_{\epsilon}\right)}{\epsilon} d x+\int_{\partial \Omega} \gamma\left(x, \phi_{\epsilon}\right) d s \\
\leq & \left|\partial \Omega_{1} \cap \Omega\right| \int_{-1}^{1}(\delta+2 f(t))^{1 / 2} d t \\
& +\left|\partial \Omega_{2} \cap \partial \Omega\right|\left|\int_{-1}^{M}(\delta+2 f(t))^{1 / 2} d t\right|+\int_{\partial \Omega_{2} \cap \partial \Omega} \gamma(x, M) d s \\
& +\left|\partial \Omega_{1} \cap \partial \Omega\right|\left|\int_{1}^{L}(\delta+2 f(t))^{1 / 2} d t\right|+\int_{\partial \Omega_{1} \cap \partial \Omega} \gamma(x, L) d s \\
= & \left|\partial \Omega_{1} \cap \Omega\right| \int_{-1}^{1}(\delta+2 f(t))^{1 / 2} d t \\
& +\int_{\partial \Omega_{2} \cap \partial \Omega}\left|\int_{-1}^{M}(\delta+2 f(t))^{1 / 2} d t\right|+\gamma(x, M) d s \\
& +\int_{\partial \Omega_{1} \cap \partial \Omega}\left|\int_{1}^{L}(\delta+2 f(t))^{1 / 2} d t\right|+\gamma(x, L) d s .
\end{aligned}
$$

Notice that the left-hand side of the above inequality is independent of $\delta, M$, and $L$; we could take the infimum with respect to these parameters on the right. Notice also the definition of $\tilde{\gamma}(x, \pm 1)$, and we get that

$$
\begin{aligned}
\limsup _{\epsilon \rightarrow 0} \inf _{\phi \in U_{r}\left(\phi_{0}\right)} I_{\epsilon}(\phi) \leq & \left|\partial \Omega_{1} \cap \Omega\right| \int_{-1}^{1}(2 f(t))^{1 / 2} d t+\int_{\partial \Omega_{2} \cap \partial \Omega} \tilde{\gamma}(x,-1) d s \\
& +\int_{\partial \Omega_{1} \cap \partial \Omega} \tilde{\gamma}(x, 1) d s \\
= & I_{0}\left(\phi_{0}\right) .
\end{aligned}
$$

We have proved the upper-bound inequality.

\section{REFERENCES}

[1] G. Alberti And A. DeSimone, Wetting of rough surfaces: A homogenization approach, Proc. R. Soc. A, 451 (2005), pp. 79-97.

[2] D. Bonn, J. Eggers, J. Indekeu, J. Meunier, And E. Rolley, Wetting and spreading, Rev. Modern Phys., 81 (2009), pp. 739-805.

[3] A. Braids, Г-Convergence for Beginners, Oxford Lecture Ser. Math. Appl. 22, Oxford University Press, New York, 2002.

[4] G. Caginalp, An analysis of a phase field model of a free boundary, Arch. Ration. Mech. Anal., 92 (1986), pp. 205-245.

[5] G. Caginalp and P. C. Fife, Dynamics of layered interfaces arising from phase boundaries, SIAM J. Appl. Math., 48 (1988), pp. 506-518.

[6] X. Chen And M. Kowalczyk, Existence of equilibria for the Cahn-Hilliard equation via local minimizers of the perimeter, Comm. Partial Differential Equations, 21 (1996), pp. 12071233.

[7] G. Dal Maso, Introduction to Г-Convergence, Birkhäuser Boston, Cambridge, MA, 1993.

[8] A. DeSimone, N. Grunewald, And F. Otto, A new model for contact angle hysteresis, Network Heterogeneous Media, 2 (2007), pp. 211-225. 
[9] E. B. Dussan, On the spreading of liquids on solid surfaces: Static and dynamic contact lines, in Ann. Rev. Fluid Mech. 11, Annual Reviews, Palo Alto, CA, 1997, pp. 371-400.

[10] P. G. De Gennes, Wetting: Statics and dynamics, Rev. Modern Phys., 57 (1985), pp. 827-863.

[11] E. Giusti, Direct Methods in the Calculus of Variations, World Scientific, River Edge, NJ, 2003.

[12] D. JACQmin, Contact-line dynamics of a diffuse fluid interface, J. Fluid Mech., 402 (2000), pp. $57-88$.

[13] H. Kusumantmaja and J. M. Yeomans, Modeling contact angle hysteresis on chemically patterned and superhydrophobic surfaces, Langmuir, 23 (2007), pp. 6019-6032.

[14] R. V. Kohn and P. Sternberg, Local minimizers and singular perturbations, Proc. Roy. Soc. Edinburgh, 111A (1989), pp. 69-84.

[15] L. ModicA, Gradient theory of phase transitions with boundary contact energy, Ann. Inst. H. Poincaré Anal. Non Linéaire, 4 (1987), pp. 487-512.

[16] J. Nevard AND J. B. Keller, Homogenization of rough boundaries and interfaces, SIAM J. Appl. Math., 57 (1997), pp. 1660-1686.

[17] R. L. Pego, Front migration in the nonlinear Cahn-Hilliard equation, Proc. Roy. Soc. London A, 422 (1989), pp. 261-278.

[18] T. QIAn, X.-P. WANG, AND P. Sheng, Molecular scale contact line hydrodynamics of immiscible flows, Phys. Rev. E, 68 (2003), 016306.

[19] D. Quere, Wetting and roughness, Annu. Rev. Mater. Res., 38 (2008), pp. 71-99.

[20] A. Turco, F. Alouges, And A. DeSimone, Wetting on rough surfaces and contact angle hysteresis: Numerical experiments based on a phase field model, M2AN Math. Model. Numer. Anal., 43 (2009), pp. 1027-1044.

[21] S. Vedantam and M. V. OAnchagnula, Constitutive modeling of contact angle hysteresis, J. Colloid Interface Sci., 321 (2008), pp. 393-400.

[22] X.-P. WAng, T. Qian, AND P. Sheng, Moving contact line on chemically patterned surfaces, J. Fluid Mech., 605 (2008), pp. 59-78.

[23] X.-P. WANG AND Y.-G. WAng, The sharp interface limit of a phase field model for moving contact line problem, Methods Appl. Anal., 14 (2007), pp. 285-292.

[24] G. Whyman, E. Bormashenko, and T. Stein, The rigorous derivative of Young, CassieBaxter and Wenzel equations and the analysis of the contact angle hysteresis phenomenon, Chem. Phys. Lett., 450 (2008), pp. 355-359.

[25] X. Xu And X. WAng, Derivation of Wenzel and Cassie equations from a phase field model for two phase flow on rough surface, SIAM J. Appl. Math., 70 (2010), pp. 2929-2941.

[26] T. Young, An essay on the cohesion of fluids, Philos. Trans. R. Soc. London, 95 (1805), pp. 65-87.

[27] P. Yue, C. Zhou, And J. Feng, Sharp-interface limit of the Cahn-Hilliard model for moving contact lines, J. Fluid Mech., 645 (2010), pp. 279-294.

Copyright (c) by SIAM. Unauthorized reproduction of this article is prohibited. 